\title{
A catalogue of omics biological ageing clocks reveals substantial commonality and associations with disease risk
}

\author{
Erin Macdonald-Dunlop ${ }^{1}$, Nele Taba ${ }^{2,3}$, Lucija Klarić4, Azra Frkatovićs ${ }^{5}$ Rosie Walker ${ }^{6}$, \\ Caroline Hayward ${ }^{4}$, Tõnu Esko ${ }^{2,7}$, Chris Haley ${ }^{4}$, Krista Fischer ${ }^{2,8}$, James F. Wilson ${ }^{1,4,}$, \\ Peter K. Joshi ${ }^{1, *}$ \\ ${ }^{1}$ Centre for Global Health Research, Usher Institute, University of Edinburgh, Edinburgh EH8 9AG, UK \\ ${ }^{2}$ Estonian Genome Centre, Institute of Genomics, University of Tartu, Tartu 51010, Estonia \\ ${ }^{3}$ Institute of Molecular and Cell Biology, University of Tartu, Tartu 51010, Estonia \\ ${ }^{4} \mathrm{MRC}$ Human Genetics Unit, Institute of Genetics and Cancer, University of Edinburgh, Western General Hospital, \\ Edinburgh EH4 2XU, UK \\ ${ }^{5}$ Genos Glycoscience Research Laboratory, Zagreb 10000, Croatia \\ ${ }^{6}$ Centre for Genomic and Experimental Medicine, Institute of Genetics and Cancer, University of Edinburgh, \\ Edinburgh EH4 2XU, UK \\ ${ }^{7}$ Program in Medical and Population Genetics, Broad Institute, Cambridge, MA 02142, USA \\ ${ }^{8}$ Institute of Mathematics and Statistics, University of Tartu, Tartu 51009, Estonia \\ *Equal contribution
}

Correspondence to: Erin Macdonald-Dunlop, James F. Wilson, Peter K. Joshi; email: e.macdonald.dunlop@ed.ac.uk; jim.wilson@ed.ac.uk; peterkjoshi2@gmail.com, https://orcid.org/0000-0002-6361-5059

Keywords: biological age, biomarkers, ageing clocks, ageing

Received: March 19, $2021 \quad$ Accepted: December 20, 2021

Published: January 24, 2022

Copyright: (c) 2022 Macdonald-Dunlop et al. This is an open access article distributed under the terms of the Creative Commons Attribution License (CC BY 3.0), which permits unrestricted use, distribution, and reproduction in any medium, provided the original author and source are credited.

\section{ABSTRACT}

Biological age (BA), a measure of functional capacity and prognostic of health outcomes that discriminates between individuals of the same chronological age (chronAge), has been estimated using a variety of biomarkers. Previous comparative studies have mainly used epigenetic models (clocks), we use $\sim 1000$ participants to compare fifteen omics ageing clocks, with correlations of 0.21-0.97 with chronAge, even with substantial sub-setting of biomarkers. These clocks track common aspects of ageing with $95 \%$ of the variance in chronAge being shared among clocks. The difference between BA and chronAge - omics clock age acceleration (OCAA) - often associates with health measures. One year's OCAA typically has the same effect on risk factors/10-year disease incidence as $\mathbf{0 . 0 9 / 0 . 2 5}$ years of chronAge. Epigenetic and IgG glycomics clocks appeared to track generalised ageing while others capture specific risks. We conclude BA is measurable and prognostic and that future work should prioritise health outcomes over chronAge.

\section{INTRODUCTION}

Age is a phenotype that we are all familiar with, and is a major risk factor for numerous diseases including the largest causes of mortality [1]. We all become acquainted with visible changes that accompany ageing, such as greying hair, baldness, loss of skin elasticity and worsening of posture, and that these vary noticeably amongst individuals of the same chronological age (chronAge). However, there are also molecular hallmarks of ageing such as telomere shortening, genomic instability and cellular senescence that also show variation in individuals of the same chronAge [1]. It has previously been hypothesised that an underlying biological age (BA), likely tagged by these molecular hallmarks, is what gives rise to age-related disease risk 
[2]. Measuring BA therefore has the potential to be more prognostic of health and functional capacity than chronAge and, as importantly, BA may be reversible [3], unlike chronAge [4].

Since this concept was proposed, there has been a push to construct models of BA, using a variety of both statistical methods and types of biomarkers; the resultant estimates we shall term omics clock ages (OCAs). The first OCAs were epigenetic clocks that used methylation levels of CpG sites across the genome - DNA methylation (DNAme) - to estimate chronAge using penalised regression [5, 6]. The excess of OCA over chronAge being omics clock age acceleration (OCAA), hopefully measuring an underlying biological effect. DNAme's verification as a meaningful $\mathrm{BA}$ measure, rather than a mere statistical artefact, was confirmed when DNAme OCAA as calculated by Horvath's clock was shown to be associated with all-cause mortality [7]. Ageing clocks trained on chronAge have since been constructed using DNA methylation $[5,6,8,9]$, telomere length $[9,10]$, facial morphology [11], neuro-imaging data [12-15], metabolomics [16], glycomics [17], proteomics [9, 1820] and immune cell counts [21]. There has however, been limited comparison of the performance, for example accuracy and correlation, of different omics ageing clocks, particularly in the same set of individuals.

As we know, second generation clocks such as DNAm PhenoAge and DNAm GrimAge have been shown to outperform previous ageing clocks by more accurately predicting mortality and health outcomes $[2,22]$. There has however, been insufficient work done to characterise the properties of ageing clocks trained on chronAge, given the number published and the fact that several have been shown to be prognostic of future health outcomes beyond chronAge [23-28]. Further characterisation of multiple omics ageing clocks trained on chronAge is essential if we want to understand what these age acceleration measures are actually capturing. Are OCA measures actually tracking underlying BA beyond chronAge or are some clocks' OCAAs more aligned to certain outcomes than others.

The deep omic and health outcome annotation of the Scottish population-based Orkney Complex Disease Study [29] cohort (ORCADES) permits interrogation of the utility and limitations of BA clocks trained on chronAge. Here, we compare the performance of 11 of our own ageing clocks built from 9 different omics assays and 4 published ageing clocks in the same set of approximately 1000 individuals in ORCADES, including whole body imaging and a clock based on the grand union of all the omics. Next, we assess the biological meaningfulness of the derived OCAA measures, by assessing their association with health- related phenotypes and incident hospital admissions (post-assessment) over up to 10 years follow-up.

The notion of BA raises fundamental questions. Is there one BA for a person, or a set of BAs, perhaps relating to different bodily systems [20, 30]. Are measured (chronAge trained) OCAs tracking a single BA, with differences arising due to their focus and accuracy, or are they tracking different underlying BAs? This study aims to shed some light on these issues.

\section{RESULTS}

\section{Performance of omics clocks}

We constructed eleven of our own ageing clocks, training on chronAge, in the ORCADES cohort from assays already understood to be able to form effective ageing clocks $[5,6,17,18]$, covering plasma Immunoglobulin G (IgG) glycans, proteins, metabolites, lipids, DNA methylation and a collection of commonly used clinical measures (such as weight, blood pressure, fasting glucose, etc.), which we label Clinomics. To this we added two novel omics sets for clock construction: a DEXA whole body imaging set of body composition measures, and one based on all the omics assays considered simultaneously, which we term Mega-omics, as listed in Table 1 (see Methods for assay descriptions).

Rather than creating completely novel DNAme clocks when effective and extensively studied published clocks exist, our methylation clocks' potential predictor sets are the subsets of the $\mathrm{CpG}$ sites used in Hannum and Horvath's epigenetic clocks available on the Illumina EPIC 850k methylation array. With this caveat, all clocks were derived from scratch using the set of available predictors and elastic net regression.

We first assessed various forms of penalised regression: LASSO, elastic net with a fixed alpha of 0.5 and elastic net with alpha calculated via cross-validation, training clocks in $75 \%$ of the ORCADES cohort and evaluating in the remaining $25 \%$ (the testing sample). We found that clock performance in estimating chronAge was independent of penalised regression method used, across all the assays (Supplementary Figure 1) and so elastic net regression with a fixed alpha of 0.5 only was employed in subsequent analyses.

To enable comparison with established omics ageing clocks trained on chronAge, we also calculated 4 clocks that have been described previously in the same $\sim 1000$ individuals in ORCADES. The four published clocks being: Hannum 2013 [5], Horvath 2013 [6], GlycanAge [17] and MetaboAge [16]. We acknowledge that DEXA-derived measures and the common clinical 
Table 1. Multiple omics make accurate ageing clocks.

\begin{tabular}{lcccc}
\hline Omic & N individuals & N predictors available & N predictors selected & r \\
\hline MetaboAge & 2019 & - & 56 & 0.21 \\
MS Fatty Acids Lipidomics & 952 & 33 & 27 & 0.45 \\
DEXA & 1158 & 28 & 28 & 0.66 \\
MS Complex Lipidomics & 940 & 908 & 130 & 0.7 \\
NMR Metabolomics & 1643 & 86 & 81 & 0.74 \\
UPLC IgG Glycomics & 1937 & 77 & 50 & 0.74 \\
GlycanAge & 2217 & - & 3 & 0.75 \\
Clinomics & 1815 & 13 & 12 & 0.8 \\
MS Metabolomics & 861 & 682 & 181 & 0.81 \\
DNAme Horvath CpGs & 957 & 333 & 155 & 0.93 \\
PEA Proteomics & 805 & 886 & 203 & 0.93 \\
Horvath 2013 & 1065 & - & 353 & 0.94 \\
Hannum 2013 & 1065 & - & 71 & 0.95 \\
DNAme Hannum CpGs & 1033 & 62 & 50 & 0.96 \\
Mega Omics & 796 & 2471 & 214 & 0.97 \\
\hline
\end{tabular}

Indicating for each omics assay: N Individuals: the number of individuals in the ORCADES cohort that passes quality control, $\mathrm{N}$ Predictors Available: the number of predictors passing assay-level quality control and therefore available for selection for inclusion in the standard model, N Predictors Selected: the number of predictors selected for inclusion in the standard model, $r$ : Pearson correlation of omics clock age (OCA) and chronAge. DEXA, Dual X-ray absorptiometry; DNAme, DNA methylation; CpG, cytosine nucleotide followed by guanine (5' to $3^{\prime}$ direction); MS, mass spectrometry; NMR, nuclear magnetic resonance; PEA, proximity extension assay; UPLC, ultra-performance liquid chromatography; IgG, Immunoglobulin G. Within each omic category, subject mean age at baseline was 53-56 (SD 15) with an age range across clocks of 16-100, whilst the proportion female ranged from 55-61\% (Supplementary Table 1).

measures (Clinomics) are not technically omics assays however, we refer to all ageing clocks as a set as omics clocks for ease from this point forward.

Ages estimated by the model in the test set (i.e. OCAs) were highly correlated with chronAge for the majority of the omics clocks tested (Table 1), particularly PEA proteomics $(\mathrm{r}=0.93)$ and DNAme based $(\mathrm{r}=0.96$ Hannum CpGs, r=0.95 Hannum 2013, r=0.94 Horvath 2013, $\mathrm{r}=0.93$ Horvath CpGs) clocks (correlations in the training set for our own clocks trained in ORCADES in Supplementary Figure 2). Unsurprisingly, the mega-omics OCA had the highest correlation ( $\mathrm{r}=0.97)$. Although all features were given equal opportunity to contribute to the mega-omics clock, those selected by the algorithm were predominantly DNAme- and PEA proteomics-based (34.6\% CpGs, 31.8\% PEA Proteomics, 20.6\% MS metabolites, $13.1 \%$ other). We found that the MetaboAge and MS Fatty Acids Lipidomics OCAs had the lowest correlations with chronAge ( $\mathrm{r}=0.21, \mathrm{r}=0.45$; Figure 1). The number of biomarkers available and then selected for model inclusion for each of our own omics clocks are indicated in Table 1 (Full list of biomarkers measured in each assay in Supplementary Table 2 and coefficients for our own clocks in Supplementary Table 3).

\section{Validation of clock performance in independent cohorts}

We next used our own clocks trained in ORCADES to estimate age in independent European cohorts to validate if they were more widely applicable beyond the Orkney population. We found that correlations between OCA and chronAge replicated to varying degrees in independent populations (Supplementary Figure 3). PEA proteomics and DNAme based clocks produced correlations of OCA and chronAge in the range of 0.89 0.98 in European cohorts replicating the range of 0.910.96 in ORCADES. UPLC IgG glycomics and Clinomics OCAs in independent populations showed a range of OCA-chronAge correlations of 0.56-0.62 compared to the 0.74-0.80 in ORCADES. Whilst the NMR metabolomics and DEXA did not replicate with correlations of $0.26-0.55$ in validation cohorts compared with 0.66-0.73 in ORCADES.

\section{Accurate performance of clocks with substantial core subset of biomarkers}

If the aim is to create BA clocks that have the potential to be clinically useful, it would be more efficient and 
cost effective to reduce the numbers of biomarkers that need to be measured in patients. To this end, we investigated the performance of our clocks using a reduced set of biomarkers. For each of our own 11 omics clocks a "core" clock was constructed using only those biomarkers which were selected for model inclusion in $>95 \%$ of 500 iterations of our clock construction procedure, as done by Enroth et al. [18] (See Methods for details). Comparable correlations of OCA and chronAge were achieved across all 11 clocks with a substantial subset or core of biomarkers (Figure 2), highlighting the potential for accurate OCAs with a small number of predictors (e.g. 30s-60s of biomarkers).

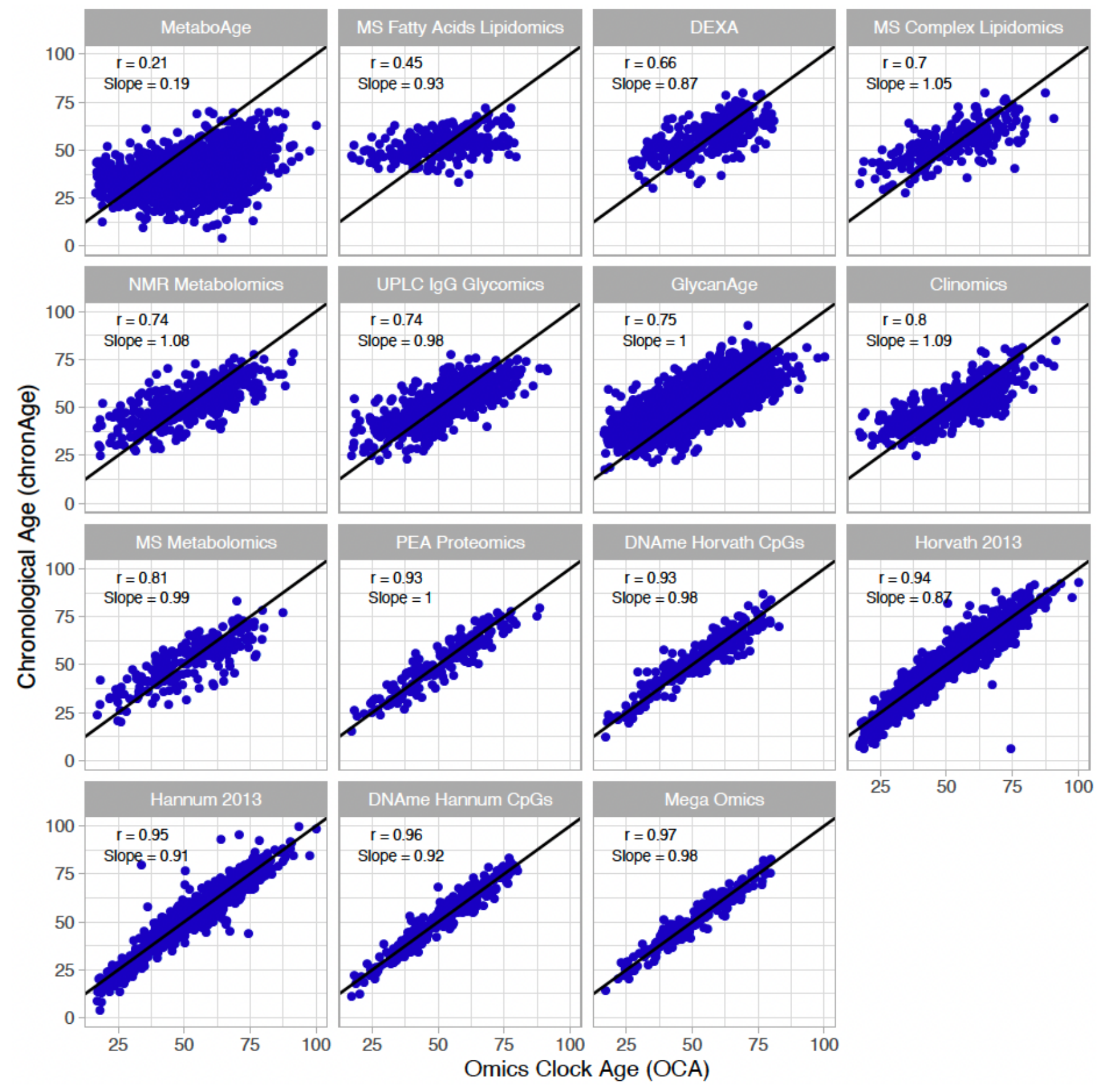

Figure 1. Multiple omics estimate chronological age, to varying degrees of accuracy, in a broadly unbiased manner. The correlations of chronAge on the $y$-axis with ages estimated by the omics ageing clock (OCA) in the ORCADES testing sample. Pearson correlation coefficient ( $r$ ) and the slope of the regression of OCA on chronAge are indicated in each panel. Identity line indicated in black. 


\section{Comparison of biological age between clocks}

Omics Clock Age Accelerations (OCAAs) showed varying degrees of positive correlation between clocks (Figure 3). Unsurprisingly, our own UPLC IgG Glycomics clock trained in ORCADES and GlycanAge were the most highly correlated OCAAs $(r=0.94)$. The four DNAme based OCAAs formed a group on their own in hierarchical clustering, with the two trained in ORCADES (Supplementary Figure 4), DNAme Hannum CpGs and DNAme Horvath CpGs OCAAs having a correlation of $r=0.73$. Within the other cluster, containing the rest of the omics OCAAs: the three clocks that are primarily constructed from lipid species and fractions, MS Fatty Acids Lipidomics, MS Metabolomics and MS Complex lipidomics, all clustered together. The DEXA, Clinomics, UPLC IgG glycomics and GlycanAge clocks formed a related group. Interestingly, the PEA Proteomics OCAA clustered on its own within in the larger non-DNA methylation cluster (Supplementary Figure 4).

For clarity, the correlations between different OCAAs and all further downstream analyses reported were performed using OCAs and OCAAs derived from the standard omics clocks as opposed to the "core" clocks described in the previous section that appear in Figure 2.

\section{Proportions of variance in age explained by different clocks}

To determine if our different clocks are explaining the same or different variance in chronAge, we partitioned the variance in chronAge explained among our clocks. We calculated the unique variance in chronAge explained by each OCA as the squared part correlations of chronAge and OCA, while controlling for all other clocks. $94.9 \%$ of the variance in chronAge is explained by two or more clocks whilst $3.6 \%$ remains unexplained by the 14 ageing clocks tested, with the remaining $1.5 \%$ being explained by one clock uniquely (Supplementary Figure 5A). The PEA proteomics and Hannum 2013 clocks explain the most variance in chronAge uncaptured by any other clock $(0.46 \%$ and $0.37 \%$ respectively; Supplementary Figure 5B). Pairwise clock comparisons are shown in Supplementary Figure 6.

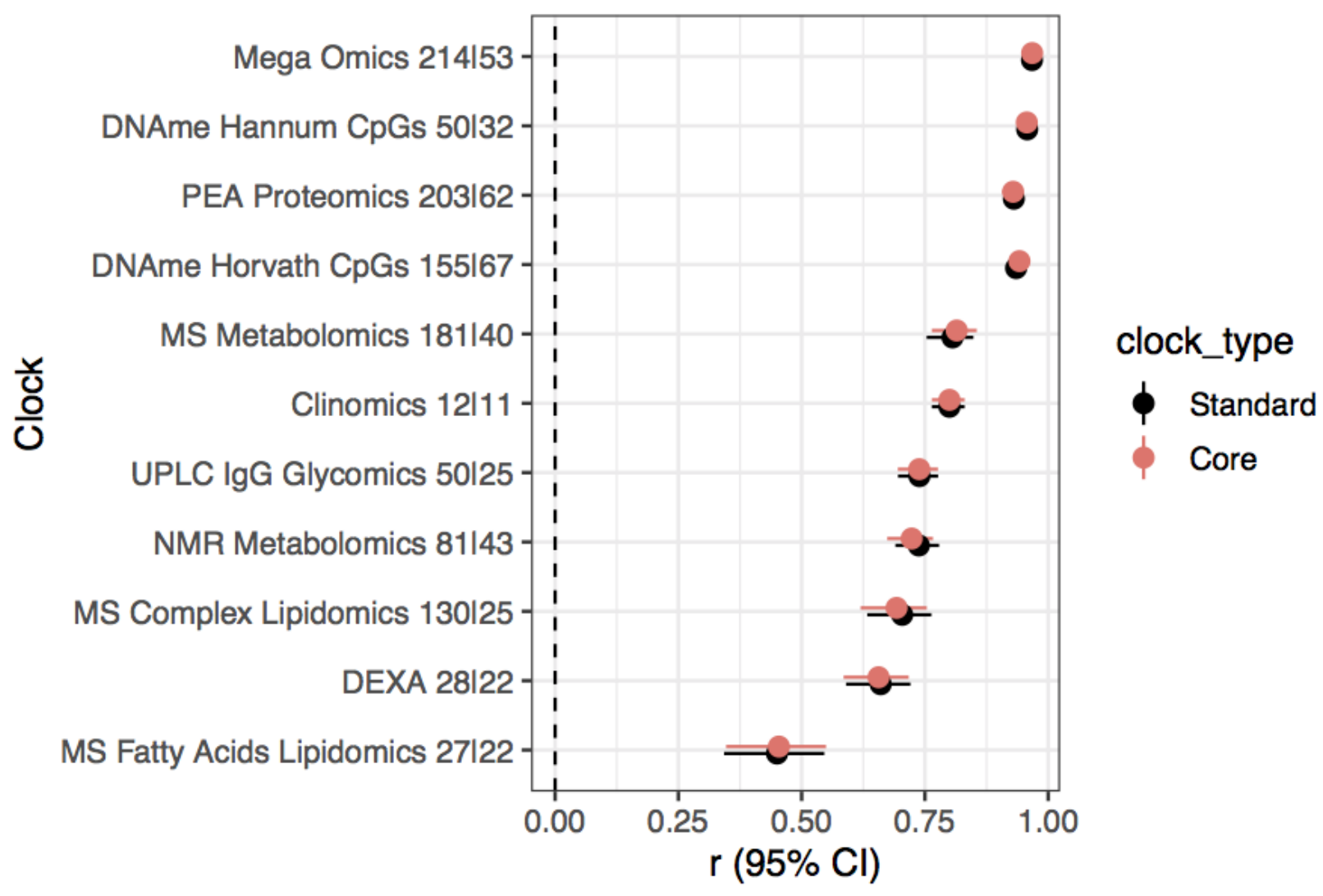

Figure 2. Substantial subsetting of biomarkers results in little dilution of accuracy. Pearson's correlation ( $r$ ) and $95 \%$ confidence interval of chronAge and OCAs from standard and core models for each omics assay indicated on the $y$-axis in the ORCADES testing sample. The number of predictors selected for inclusion in the standard and then core models are indicated in the $y$-axis labels (standard|core). 
Having found that clocks overlap in the information they provide about chronAge, we tested to see if, together, pairs of clocks jointly explained a different proportion of variance in chronAge than would be expected if the clocks were each independently sampling from a latent set of complete predictors of chronAge (ISLSP). This analysis should reveal whether the clocks were tracking complementary dimensions of ageing: situations where the pair of clocks overlapped less than expected if they were independently sampling (negative values on this scale). Strikingly, excess overlap was found across all pairs of clocks bar MS
Fatty Acid Lipidomics and MetaboAge (Figure 4). All other pairs had excess overlap values >0.16 (comparison of the MetaboAge and DEXA clocks), suggesting that these clocks, considered pairwise, track more common rather than complementary aspects of chronAge.

Unsurprisingly, the most overlapping were our own UPLC IgG Glycomics clock trained in ORCADES and GlycanAge (excess overlap of 1; note on our scale, a clock shows 1.00 excess overlap with itself, whilst ISLSP would show 0.00) indicating they are capturing

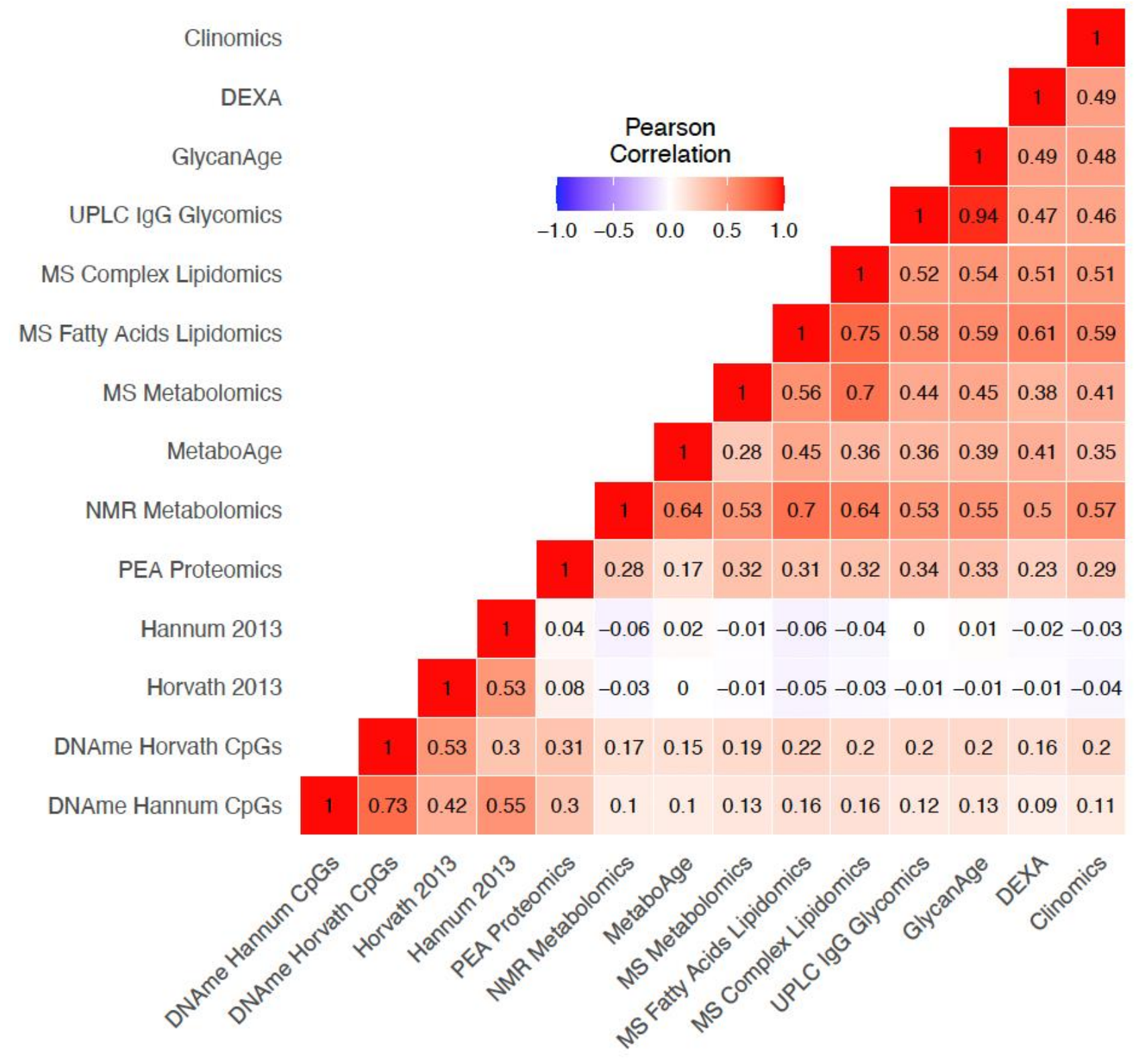

Figure 3. Variable positive correlations between different omics age accelerations. Pearson correlation of OCAAs (omics clock agechronAge) in ORCADES testing and training samples. Colour indicates the direction and the shade and number indicate the magnitude of the correlation. Rows and columns are ordered based on hierarchical clustering of the pairwise correlations. 
entirely overlapping variance in age. These Glycomicsbased clocks showed high excess overlap with the PEA Proteomics clock (0.95 and 0.96). The MS Fatty Acids Lipidomics, MS Complex Lipidomics and MS Metabolomics clocks showed high excess overlap with each other (0.97-0.98). Interestingly, the NMR Metabolomics clock, which also contains many lipid features, did not cluster with these three clocks. The four DNAme-based clocks clustered tightly together with DNAme Hannum CpGs and DNAme Horvath CpGs having an excess overlap of 0.91. As these clocks are extremely accurate chronAge predictors, a large amount of overlap in variance explained is inevitable; they are tracking common aspects of ageing. Interestingly, MetaboAge clustered on its own and showed higher excess overlap with DNAme- and PEA Proteomics clocks than those based on metabolite or lipid measures.

\section{Excess Overlap $\quad 0.00 \quad 0.25 \quad 0.50 \quad 0.75 \quad 1.00$}
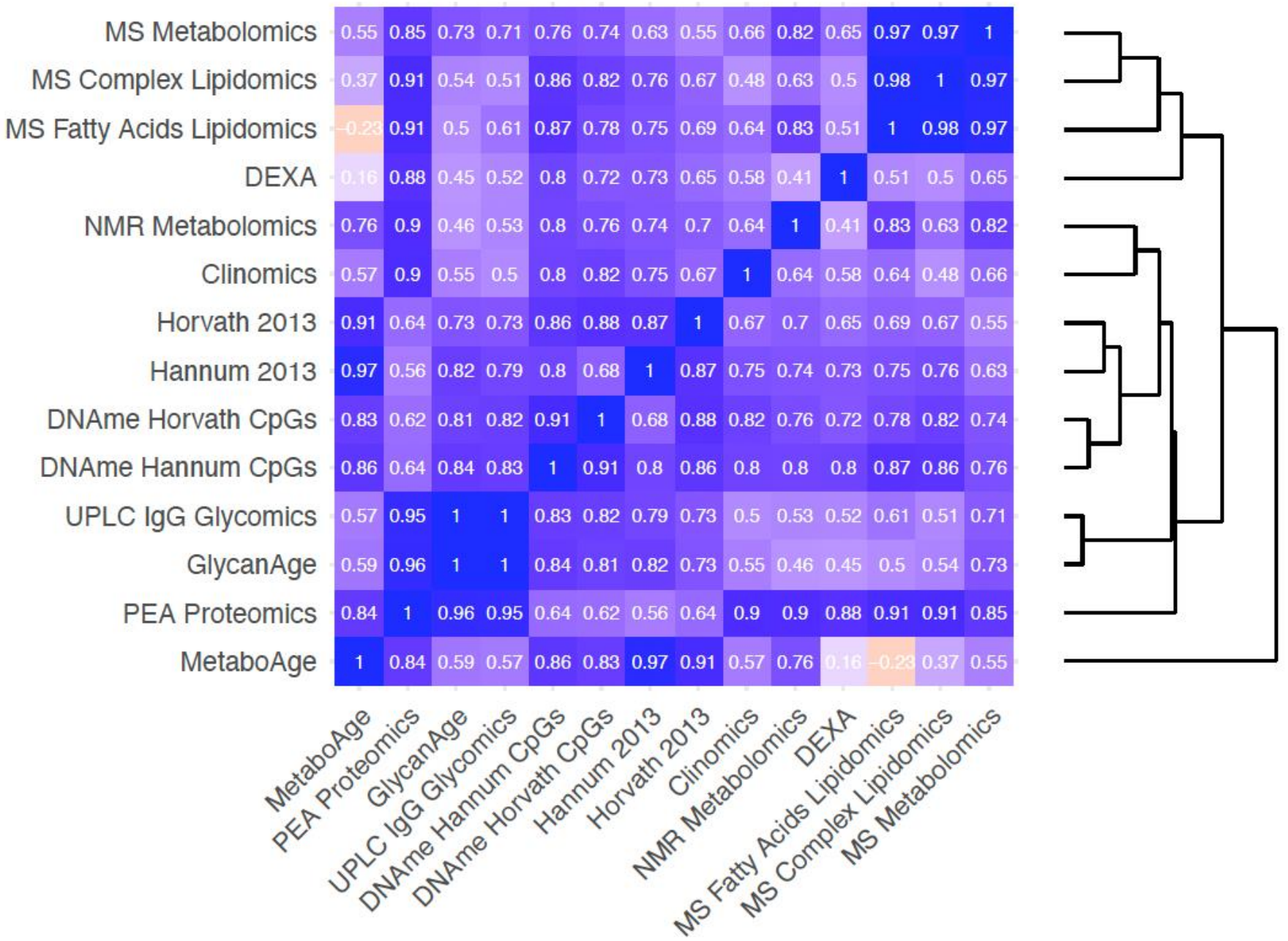

Figure 4. Bivariate analyses reveal that clock pairs tend to overlap more than expected by chance in the variance in chronAge they explain. The amount of excess overlap that would be expected by chance is indicated for each pair of clocks. This is the deviation of the observed variance in chronAge explained by a bivariate model containing a pair of OCAs and the variance expected to be explained by that pair given that we know how much variance in chronAge they explain individually, if each of the clocks were independent samples from a set of latent complete predictors. This measure of deviation of observed from expected is scaled (See Methods for details) so that a value of 1 means that the second clock is adding no more information than the first, meaning that they overlap entirely in the information they provide about chronAge. A value of 0 would indicate the observed variance explained in chronAge is exactly what is expected if the two clocks were independently sampling. Negative values ind icate disproportionately complementary components of chronAge were being tracked. 


\section{OCAAs compared to chronAge as predictors of disease risk}

We next sought to test the effect of OCAAs compared to chronAge on risk factors and post assessment disease incidence, as measured by hospitalisation in the ORCADES cohort, where the outcome was thought $a$ priori to associate with age. For risk factors we chose body mass index (BMI), systolic blood pressure (SBP), cortisol, creatinine, C-reactive protein (CRP), forced expiratory volume in 1 second (FEV1), and total cholesterol. For diseases we chose five International Statistical Classification of Diseases and Related Health Problems (ICD)-10 Chapters: II (Neoplasms - codes C), IV (Endocrine, nutritional and metabolic diseases codes E), IX (Diseases of the circulatory system - codes I), and X (Diseases of the respiratory system - codes J). The ICD-10 blocks used and their coding titles are listed in Supplementary Table 4).

In order to compare OCAA and chronAge, we first quantified the effect of chronAge on risk factors and disease (Supplementary Figure 7A, 7B). All 7 risk factors and 32/44 disease blocks were taken forward as they were significantly associated with chronAge (beta $>0, \mathrm{FDR}<10 \%$ ) and had $>5$ incident cases (disease blocks). The effect of chronAge on (standardised) risk factors appeared to vary by trait, whereas for diseases, it appeared that the effect of chronAge (on the hazard ratio scale) might be similar across diseases, with a consistent doubling of risk every 14 years.

We tested for risk factor and disease associations with OCAA, using chronAge and sex as covariates. Results were then rescaled to be per year of chronAge effect by dividing the observed effect of OCAA by the effect of chronAge on the outcomes as identified at the previous step. This was taken trait-by-trait for risk factors, and a single effect for all disease groups and chapters: -0.0492 $\log _{\mathrm{e}} \mathrm{HR}$.

Despite limited power for detecting OCAA-disease associations, 6/480 tests were statistically significant $(\mathrm{FDR}<10 \%)$ as were $19 / 90$ OCAA-risk factor associations. We also found evidence of enrichment of positive effects of OCAA on both risk factors $(81.1 \%)$ and disease (73\%), with $43.3 \%$ and $22.3 \%$ being nominally significant (one sided $\mathrm{p}<0.05$ ), respectively. Across clocks, the inverse variance-weighted mean effect of one year of OCAA on risk factors/disease was the same as 0.09/0.25 years of chronAge (SE 0.01/0.02, note here and elsewhere $\sim$ denotes indicative, see Methods for details). Interestingly, the mean effect across all diseases of one year's DNAme Hannum/ Horvath CpGs OCAA was similar to one year of chronAge (ratio: 1.03/0.85, SEs $\sim 0.18$ ), but the effect on risk factors was much lower (ratio: 0.06/0.17, SEs 0.09). Complete results are shown in Supplementary Table 5 and inverse variance-weighted effects are shown in Supplementary Figure 8A.

In general, only associations with the Clinomics OCAA passed FDR, however DNAme Hannum CpGs OCAA, our own UPLC IgG Glycomics OCAA trained in ORCADES and GlycanAge were nominally associated with twelve ICD10 blocks, one more than Clinomics and DNAme Horvath CpGs OCAA (Figure 5). In contrast, the PEA proteomics clock ( $\mathrm{r}=0.93$ with chronAge) showed only one nominally significant disease-OCAA association. Looking at disease groupings, E70-E90 Metabolic disorders and J09-J18 Influenza and Pneumonia showed the most nominal associations across all OCAAs. Curiously, on the other hand, C34-C44 Melanoma and C51-59 Malignant Neoplasms of the female genital organs, showed generally negative associations with OCAAs.

For the analysis of the effect of OCAA on risk factors, Clinomics was excluded as its predictors (e.g. cholesterol, FEV1 and SBP) are often close to and designed to predict clinical endpoints and overlap with the risk factors considered here. Similarly, as the NMR Metabolomics assay contains a measure of creatinine, we excluded the association of both our own NMR Metabolomics OCAA trained in ORCADES and MetaboAge with this outcome. Finally, for the same reason we excluded the association of total cholesterol with NMR metabolomics, MetaboAge, MS Metabolomics, MS Complex Lipidomics and MS Fatty Acid Lipidomics OCAAs.

The greater statistical power for risk factors results in considerably more significant associations at FDR $<10 \%$ (Figure 6). DEXA and UPLC IgG Glycomics OCAAs were associated with the most risk factors (4 at 10\% FDR), with Mega-omics, MS and NMR Metabolomics OCAAs showing positive associations with all risk factors. All OCAAs tested were associated positively with BMI and total cholesterol. We found strong associations between OCAAs and the marker of inflammation CRP (often with effect sizes $>1$ ), meaning OCAA had a larger effect than chronAge. Overall, the averaged effect of OCAA on risk factors as a proportion of the effect on diseases was large for PEA proteomics and Mega-Omics (463\% and $81.2 \%$ respectively) suggesting they are directly tracking the risk factors we considered. Conversely, this proportion was small for DNAme Hannum CpGs, DName Horvath CpGs, Hannum 2013, Horvath 2013, MS Fatty Acid Lipidomics, UPLC IgG Glycomics and GlycanAge $(6.3 \%, 19.3 \%,-0.7 \%,-6.5 \%,-0.5 \%, 33.4 \%$ and $39.4 \%$ respectively), suggesting they are prognostic of incident 
disease and therefore track more generalised ageing (Supplementary Figure 8B).

We wanted to check if observed OCAA-health associations were driven by the associations of health with smoking and of OCAA with smoking. Our analysis fitting smoking status as a confounder suggests they were not (Supplementary Figure 9A, 9B).

\section{Comparison of predictive abilities of different OCAAs for risk factors and disease}

In principle, two OCAAs could have the same association effect size on disease, but one might be much more prognostic for the population as a whole than the other if it had much larger variation in its range. In order to determine which OCAAs could draw more meaningful distinctions between subjects in terms of health outcomes, we repeated the previous analysis using standardised OCAAs. Standardised OCAAs on risk factors had a narrow range, standardised NMR Metabolomics OCAA showed the greatest predictive power, with an IVW-average effect across all risk factors of 0.09 (SE 0.01). Other clocks ranged from 0.07-0.03, with Hannum 2013 and Horvath 2013, MetaboAge and MS Fatty Acid Lipidomics OCAAs smaller still, spanning -0.01-0.01 (SEs 0.01, in all cases). Conversely, CRP and total cholesterol were

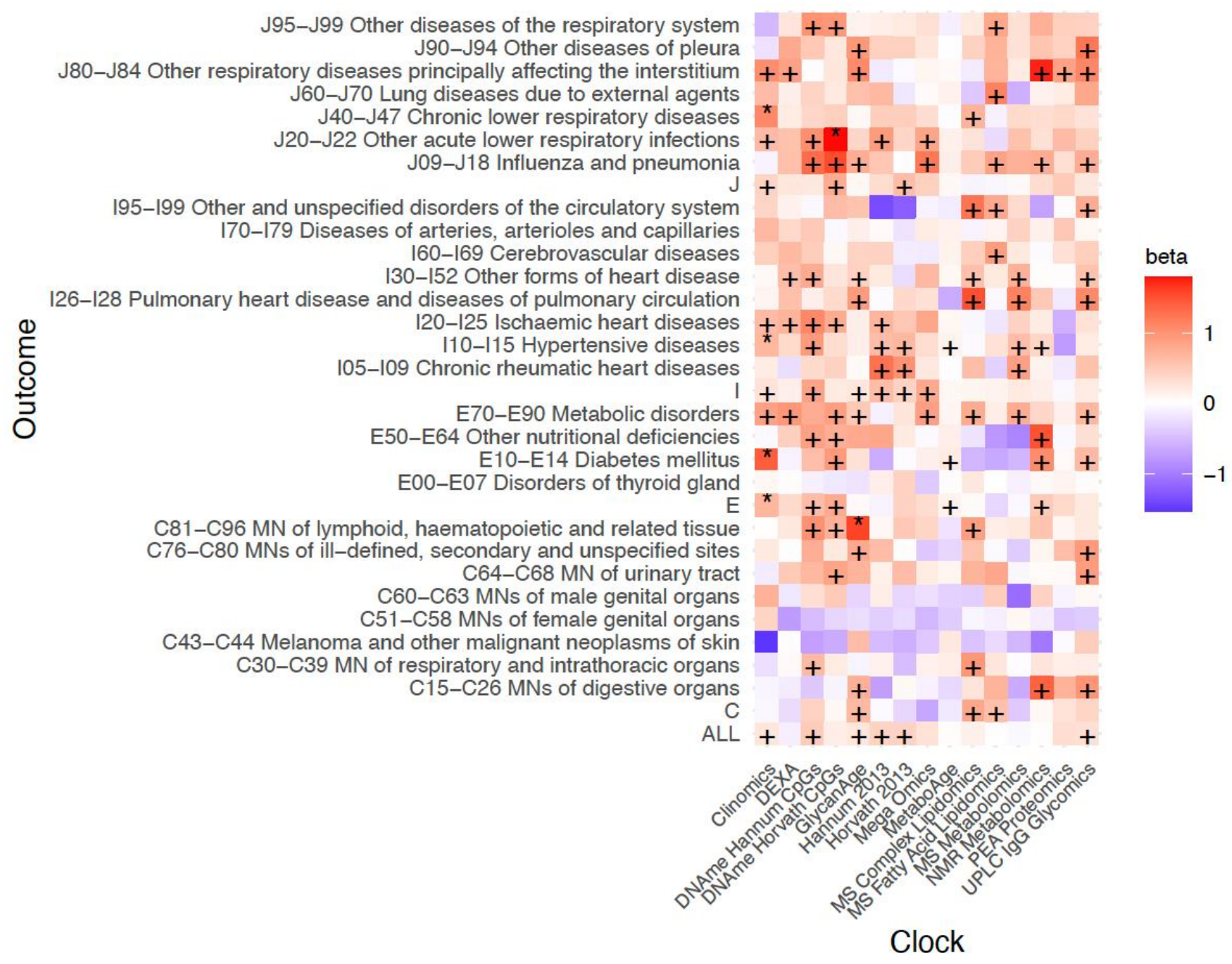

Figure 5. Positive age acceleration associations observed with increased disease risk. Associations with rates of hospitalisation. $+/ *$ Association nominally/FDR $<10 \%$ significant in the frequentist test that OCAA has a positive effect on outcomes. Beta: the relative effect of a year of OCAA to a year of chronAge on disease (initially measured in $\log _{\mathrm{e}}$ hazard ratios, effect sizes are unitless after division). A value of one indicates that a year of OCAA is equally as deleterious as a year of chronAge and is indicated in salmon colour. To facilitate reading, note the DNAme Horvath CpGs-BMI beta is 1.02 and the DNAme Hannum CpGs-C81-C96I beta is 1.00. Clock: the omics clock on which OCAA was measured. Disease group: the set of diseases (defined by ICD10 codes) which were tested for first incidence after assessment against the clock, already prevalent cases were excluded (Case numbers for each disease block in Supplementary Table 5). 
most predictable by standardised OCAA $(0.08$ and 0.06 , SEs $\sim 0.01$, IVW-averaged across clocks), whilst systolic blood pressure was least predictable (0.02, SE 0.01).

Standardised OCAA effects on disease showed a less uniform pattern (Supplementary Figure 8B): the IVWaverage effect across diseases was between 0.09 (Hannum 2013) and 0.24 (Clinomics), except for the 0.017 and 0.026 of the PEA Proteomics and Horvath 2013 respectively (SEs $\sim 0.04$ and $\sim 0.03$ ). Despite limited power, the disease group showing the most sensitivity to standardised OCAA across clocks was J80-J84 (Other respiratory diseases principally affecting the interstitium; 0.56, SE 0.13).

We were interested to see if the effect of our OCAAdisease associations were consistent across the sexes. To mitigate issues with sample size, we restricted analysis to OCAA-disease block associations which were nominally significant $(\mathrm{p}<0.05)$ in the pooled analysis. Of the 107 nominally significant associations from the pooled analysis, 78 passed the criteria (significantly associated with chronAge, beta $>0$, FDR $<10 \%$, and $>5$ cases) to be assessed in each sex separately (Supplementary Table 6). Of these 78 OCAA-disease associations, the sign of the effect was consistent across the sexes $92.3 \%$ of the time. For those $(n=6)$ where the sign was discrepant, we performed a two-sided t-test for a statistically significant difference in standardised effect size, essentially checking whether the discrepancy is plausibly explained by chance. None showed a difference at a nominal level $(\mathrm{p}<0.05)$.

\section{Clocks built from few omics principal components are effective predictors of health outcomes}

Finally, we reduced dimensionality and assessed the underlying information about ageing being captured by different omics at the assay level, rather than simply the predictors selected for model inclusion. We constructed versions of our own clocks trained in ORCADES using a few principal components (PCs) of omics measures as predictors and repeated the previous analyses with their (standardised) OCAAs, estimating chronAge (Supplementary Figure 10) and predicting health outcomes (Supplementary Figure 11A, 11B). The pattern was striking, the IVW-mean effect sizes across all risk factors of 3 PC OCAAs were more than double

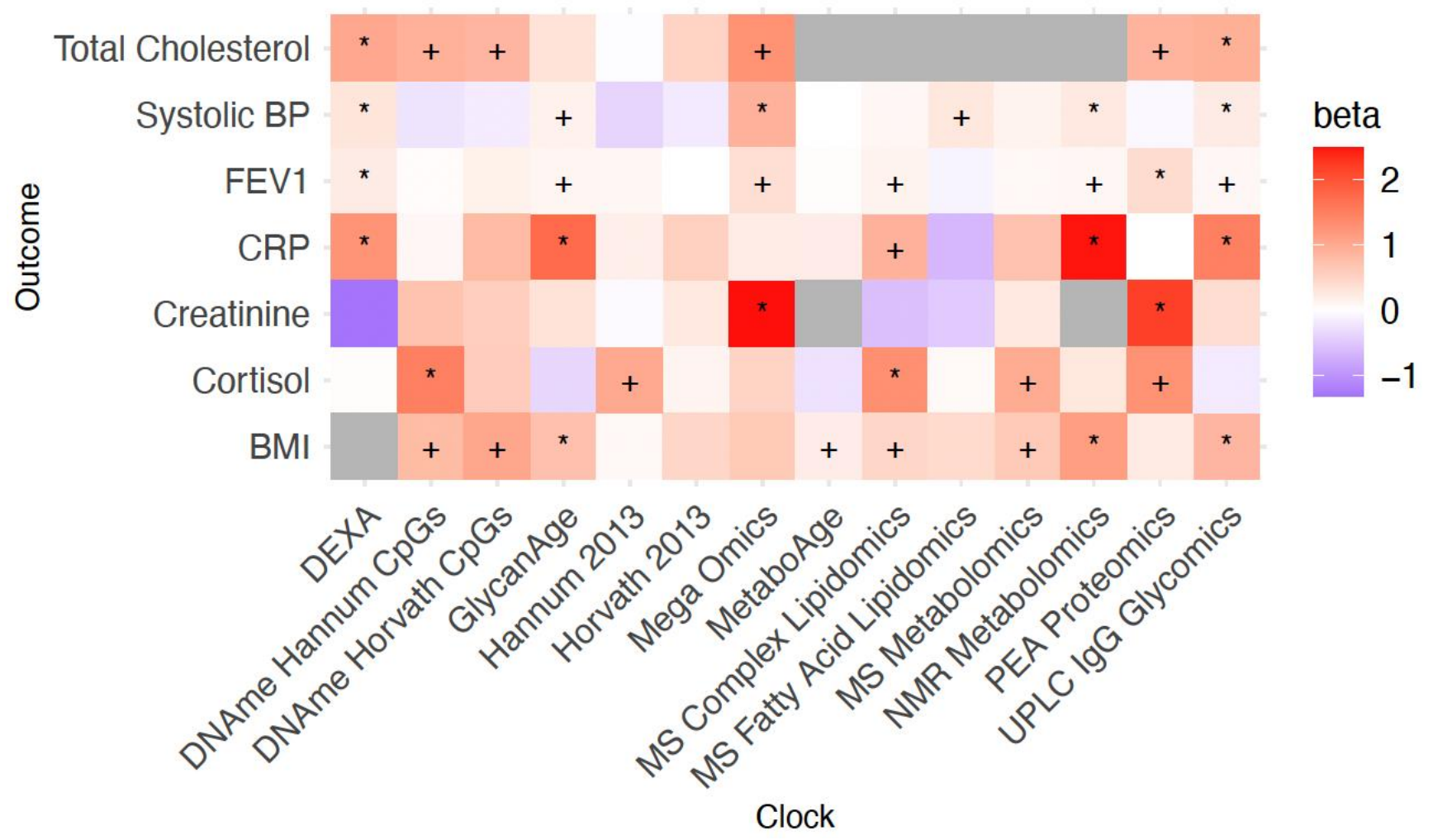

Figure 6. Positive age acceleration associations observed with increased disease risk. Associations with disease risk factors. $+/ *$ Association nominally/FDR $10 \%$ significant in the frequentist test that OCAA has a positive effect on Risk factors. Beta: the relative effect of a year of OCAA to a year of chronAge on risk factor (effect sizes are unitless after division). A value of one indicates that a year of OCAA is equally as deleterious as a year of chronAge and is indicated in salmon colour. 
our standard OCAAs (Supplementary Figure 11A). For all OCAAs, bar DNAme-based, including more omics PCs in the clocks reduced their ability to estimate distinctions in risk factors. IVW-mean effects on diseases were generally similar for the $3 \mathrm{PC}$ and standard OCAAs, except for the PEA Proteomics OCAA, where 3 PCs- based clock outperformed the standard clock by a factor of 10. Overall, OCAAs derived from a few omic PCs appeared equally predictive as our standard OCAAs for diseases and more predictive for health risk factors.

\section{DISCUSSION}

We have performed the most exhaustive comparison of different omics assays as potential biomarkers of age to date. We have shown firstly, it is possible to construct ageing clocks that produce highly accurate estimations of chronAge with a wide variety of omics biomarkers. Secondly, our own ageing clocks built using PEA proteomics, DNAme, UPLC IgG glycomics and clinical risk factors in ORCADES were able to estimate chronAge in independent populations. Thirdly, it is possible to achieve the same highly accurate estimation of chronAge using a substantial subset of core biomarkers from each assay. Despite finding only modest positive correlations between our OCAAs, we showed that different clocks overlap in the variation they explain in chronAge more than would be expected by chance if they were independently sampling from a latent set of complete predictors. We found associations of OCAAs with total cholesterol, C-reactive protein, BMI, creatinine, cortisol, FEV1 and systolic blood pressure. We found 6 statistically significant $(\mathrm{FDR}<10 \%)$ individual associations and strong evidence of enrichment of association of OCAA with incident disease collectively across our tests $(22.3 \%$ were nominally significant $\mathrm{p}<0.05$ ). We found Less variation in OCAA predictiveness across risk factors, than across diseases. Overall, we estimated that one year of OCAA has an effect of 0.09/0.25 years of chronAge on risk factors/disease incidence and showed that OCAA based on clocks built using a few principal components of omics were as prognostic as those presented with all available features.

The correlation of our PEA proteomics, DNAme, UPLC IgG glycomics OCAs and chronAge were similar to correlation coefficients reported with published models $[5,6,17,18]$. Comparisons within ORCADES, of our own and published clocks, showed that correlations with chronAge were consistent across IgG Glycomics and DNA methylation assays, however our own NMR Metabolomics OCA had a higher correlation with chronAge $(\mathrm{r}=0.74)$ than MetaboAge $(\mathrm{r}=0.21)$.
Unsurprisingly, DNAme-based clocks built in ORCADES were able to estimate age in both Scottish (Generation Scotland) and Estonian Biobanks (EBB), as the Hannum 2013 and Horvath 2013 epigenetic clocks have been used successfully in numerous populations. We showed for the first time that clocks built from Olink PEA-based proteomics replicate (in EBB and Croatia-Vis), while clocks built using the SOMAlogic [20] proteomics platform have been shown to replicate across populations previously. Our UPLC IgG glycomics clock also replicated in an independent population, mirroring the applicability of published GlycanAge measures [17]. Conversely, our NMR metabolomics and DEXA clocks had much lower correlation with chronAge in EBB and UKB. The success of these clocks appears to be study-specific: differences in lifestyle and environmental factors that change with age between the populations of the Orkney Islands and general populations in the UK and Estonia are a plausible cause. Interestingly, while MetaboAge has been shown to replicate with consistent correlation of OCAA and chronAge in two populations $(\mathrm{r}=0.65$ and $\mathrm{r}=0.70)[9,16]$, it showed a considerably lower correlation in ORCADES, $r=0.21$. These findings serve as a warning as to the generalisability of ageing clocks to new populations.

For a measure of BA to be clinically useful and efficient, effective age estimation based on as few predictors as possible is ideal. We substantially reduced the numbers of biomarkers from each assay that were included in our clocks and showed no dilution of performance across all of our own clocks. Enroth et al. [18] showed that this was possible with a protein-based clock, however, we reduced the number of proteins by a larger factor and achieved the same accuracy estimating chronAge. This high performance with a substantial subset of predictors has not previously been shown systematically across nine different types of biomarkers.

The extremely high correlations with chronAge reported, such as the $\mathrm{r}=0.97$ of the Mega-omics OCA, highlight an issue that has been discussed in prior work: that if enough biomarkers were included in the model it would be possible to perfectly estimate chronAge and, by definition, fail to detect (distinct) BA. Lehallier et al. [20] showed that correlation between OCA and chronAge increases with the number of proteins included in the model. Further, it is possible to explain $100 \%$ of the variance in chronAge using DNAme data in large samples [31]. A perfect age predictor would give no information about variation between individuals of the same age and even those which are near perfect will have too little variation in the OCAA to be indicative of health status or outcomes beyond chronAge [32]. We found this trend in our results, that 
the most accurate estimators of chronAge: Mega-omics and PEA proteomics OCAAs were not strongly associated with subsequent hospital admissions, nor DNAme-based OCAAs with risk factors. Of course extremely accurate estimators of chronAge do have their uses, for example in a forensic context [33], but are not useful in terms of BA. This does not mean the assays themselves cannot be used to estimate BA but highlights a limitation of training ageing clocks on chronAge.

A useful BA must be an indicator of health status or outcomes beyond chronAge. We found DNAme-based OCAAs were better estimators of incident disease than risk factors, consistent with the known performance of the Horvath 2013 epigenetic clock. Several groups have shown Horvath 2013 OCAA to be associated with subsequent all-cause mortality [7, 34-37]. Differences in Horvath 2013 OCAA between cases and controls have been found for numerous disease phenotypes [34, 38-47]. In contrast, Horvath 2013 OCAA has been found not to be associated with common risk factors including: LDL cholesterol and CRP [28], a finding we confirmed. We found that PEA Proteomics and Megaomics OCAAs were better at predicting risk factors than disease, whereas the opposite was true for DNAme- and IgG Glycomics-based OCAAs. In contrast, DNAmeand IgG Glycomics-based OCAAs being prognostic of incident disease beyond chronAge suggests they are more likely to be capturing underlying BA.

It is perhaps not surprising that the Clinomics OCAA showed the strongest evidence of association with disease - it used common clinical measures thought to be prognostic of health. Nonetheless, the pattern is a reassuring proof of concept. The overall enrichment of OCAA-disease and -risk factor associations, strengthens the case for the notion of BA, trackable through omics markers.

Jansen et al. [9] showed that MetaboAge was significantly higher in cases of metabolic syndrome and cardiometabolic disease than controls, however was not prognostic of incident disease. In contrast, van den Akker et al. found MetaboAge to be associated with increased risk of all-cause mortality, coronary and cardiovascular events [16]. Despite limited power, our results align with the latter, we found MetaboAge OCAA in ORCADES to be nominally associated $(\mathrm{p}<0.05)$ with incident diabetes (E10-E14), hypertensive disease (I10-I15) and group E ICD10 codes (Figure 5). Similarly, our own NMR Metabolomics OCAA was nominally associated $(\mathrm{p}<0.05)$ with several metabolic disease blocks, suggesting that in a more powered sample this relationship would be significant. Previously, it has been shown that GlycanAge is associated with risk factors [17] and that IgG glycans (i.e. not an OCAA, rather the glycan levels themselves) are effective predictors of incident type 2 diabetes and cardiovascular events [48-50]. However, we are the first to show IgG Glycan-based clocks (GlycanAge and our own UPLC IgG glycomics OCAA) to be prognostic of incident disease and highlight this is not simply due to tracking the risk factors we considered.

MetaboAge stood out in several analysis: its low correlation with chronAge, subsequent low unique variance explained in chronAge and low overlap of variance explained in chronAge in pairwise comparisons across all other clocks suggest that MetaboAge may not be tracking chronAge. These combined with the nominal associations of MetaboAge OCAA with incident cardiometabolic outcomes despite limited power, add support to the argument that omics ageing clocks should be more outcome focussed and move away from training on chronAge.

As by definition, having a BA of +1 indicates that the individual has the same functional capacity and risk of age-related disease as the average individual that is one calendar year older than them, indicating the effect of true BA is the same as 1 year of chronAge. Our estimate that the mean effect of 1 year of OCAA on disease incidence is the same as 0.25 years of chronAge is important. BA thus appears to be real and measurable and have effects of similar magnitude to chronAge, albeit our estimates are significantly diluted compared to chronAge, possibly due to OCAA capturing only some aspects of BA, reflecting the types of assay and tissue, rather than BA itself. Better measures of BA seem worthy of pursuit, as do interventions that can reverse well-measured BA. The negative association between Melanoma and other malignant neoplasms of skin (C43-C44) and OCAAs for many clocks leads us to speculate that a less sedentary lifestyle is leading to lower OCAA, but also increased exposure to the sun. If replicated, this will highlight that skin BA and other BAs need not closely align, and we speculate this finding might also generalise across other organs.

A strength of our work was the sheer number and range of assays and therefore omics ageing clocks whose performance we compared in the same individuals, whereas previous comparisons have been limited to DNAme-based clocks [23, 24, 51] or DNAme, clinical risk factors and frailty measures [25]. We also directly compared our own omics ageing clocks trained in the ORCADES cohort with published clocks. We have tried to validate our omics ageing clocks trained in ORCADES in independent populations where available, to illustrate their wider applicability. A limitation faced by previous studies was the narrow age range of 
individuals in the training sample, for example Lee et al.'s epigenetic clock trained in a pregnancy cohort produced extremely accurate estimations of chronAge for individuals under 45 but underestimated age in older individuals [52]. Our clocks avoid this limitation due to the wide age range (16-100) of individuals in the ORCADES cohort.

The novel assessment of excess overlap between clocks is a strength of this work, as it has not previously been shown that, across multiple different omics assays, OCAs overlap more than would be expected by chance if they were ISLSP, indicating these clocks are tracking more common rather than complementary aspects of ageing. A further strength is the regularisation of effect sizes - we have measured the effect of OCAA per effect of year of chronAge - giving a natural and understandable scale. Another strength is its scope, with many clocks tested against many age-related diseases. Of course, this is also a weakness, as it reduces power after compensation for multiple testing. Nonetheless, the essentially agnostic view taken of individual disease groupings and clocks does mitigate the risk of publication bias.

A limitation of this work is the relatively small sample size, both in terms of the number of individuals with multiple omics assays and within that, the number of incident hospital admissions over the follow-up period. Due to the low number of deaths in our sample we are as yet unable to test for the association of OCAA on mortality, as in previous studies. The issue of sample size combined with the exacerbation of multiple testing limited our power to assess the effect of OCAA measures on subsequent incident disease over and above common risk factors as in previous studies [7, 25], however, this should be investigated in larger samples. We did however investigate whether the effect of OCAAs on incident disease differed across sexes. To maximise power and limit multiple testing we restricted this analysis to the associations that were nominally $(\mathrm{p}<0.05)$ significant in the pooled analysis and that passed the same criteria (beta>0 FDR $10 \%$ one sided association with chronAge and $>5$ cases). We did not find evidence of significant sex differences.

As our aim was to characterise the properties of ageing clocks trained on chronAge, derived from a wide range of omics assays, in order to understand what these measures are actually capturing as this has been understudied, we did not compare our clocks with those trained on mortality-based measures [2, 22]. A study focussed on systematically comparing the utility of chronAge versus mortality-based clocks could be undertaken, however that was not our aim.
As the omics data available for ORCADES is crosssectional, we were unable to comment on the variation of OCAA within individuals over time. However, we were able to investigate the prognostic ability of single time point OCAAs on hospital admissions over a 10year follow up. While not explicitly testing OCAAs for their association with healthspan as defined by Zenin et al. [53], by including the ALL (first diagnosis in any of the disease blocks considered) category in our analysis, we have assessed an equivalent measure of risk of major disease. The key differences are that we have not included dementia, as incidence is very low, nor have we included death (as mentioned above), noting that Zenin et al. observed death as defining healthspan in less than $2 \%$ of cases. We have however included more diseases, all of which we have shown are age related.

The nature of our sample, a population isolate, means there is potential for local factors to influence our results. We have shown this is not the case for several of our omics clocks' accuracies (Supplementary Figure 2 ), as they were successfully replicated in additional populations, however, it could contribute to the poor replication seen for the DEXA and NMR metabolomics clocks. The use of hospitalisation as a measure of incidence is a limitation, particularly acute for diseases normally treated in the community such as type 2 diabetes and influenza. Nonetheless, we are likely to have captured the most severe cases and have tested whether this severity associates with OCAA and presumed frailty, giving rise to more severe experience of the disease. Secondly, the correlated nature of the assays and of the disease outcomes mean our tests have not been independent, although this means the FDR corrections have been conservative. A more powered study might also try to disentangle individual markers especially those retained in our core omics clocks and consider their biological plausibility as sitting on the causal pathway.

Of course, association does not imply causation. Although the use of a prospective cohort has reduced the risk of reverse causation, undiagnosed cases (at baseline) might still have contributed to the effects we observe, although confounding where a latent set of underlying traits is influencing disease susceptibility and the biomarkers is perhaps more likely. Nonetheless, even in the absence of causation, OCAA does appear to often be a biomarker of disease and underlying BA.

In conclusion, our work has strongly further evidenced the existence of BA as distinct from chronAge, whilst highlighting a substantial part of the OCAA is noise. The data also suggested there may be more than one type of BA, as measured by different clocks and giving rise to differing amounts of disease susceptibility, 
most strongly implied by our evidence that skin age and heart age may move in opposite directions. We also highlight that some OCAAs (e.g. PEA proteomics) may capture specific risks and consequently associate with health, whilst others (e.g. DNAme- and IgG glycomics-based) may capture more generalised ageing. Our observation that clocks derived from few PCs of omics are less accurate in estimating chronAge but better able to predict risk factors, suggests that the search for BA should be pursued through salient features of biology. This supports the recent success of ageing clocks trained on all-cause mortality based measures [2, 22], DNAme PhenoAge [2] and GrimAge [22], which have been shown to be more prognostic of health and mortality outcomes than DNAme clocks trained on chronAge directly [24-26, 54]. Similarly, the mortality trained NMR Metabolomics measure from Deelen et al. is more prognostic of both 5- and 10-year allcause mortality than a model of conventional mortality risk factors [55]. We therefore suggest that the focus of future research should continue to shift to clocks trained on mortality, or more ideally all-cause morbidity, that are prognostic of subsequent health outcomes rather than accurate chronAge estimators.

\section{MATERIALS AND METHODS}

\section{Cohort data}

Analyses were predominantly carried out using the Orkney Complex Disease Study (ORCADES) [29], a population-based isolate cohort that is extensively characterised in terms of both traditional phenotypes, omics assays and mean 12 years of follow up via linked electronic health records (EHR). The additional cohorts, Croatia-Vis and Croatia-Korčula [56, 57], were used to validate omics ageing clocks trained in ORCADES. Croatia-Vis was used to validate a clock trained in ORCADES using a subset of proteins (those measured on the Olink CVDII, CVDIII and INFI panels) referred to as protein subset 1 and the UPLC IgG glycomics clock. Replication of the NMR metabolomics and UPLC IgG glycomics clocks trained in ORCADES was carried out in Croatia-Korčula. The Estonian Biobank [58] (EBB) cohort was used to validate a clock trained using a subset of proteins (those measured on the Olink CVII, CVDIII, INF1 and ONCII panels) referred to as protein subset 2 as well as the NMR Metabolomics clock. Both EBB and the Generation Scotland: Scottish Family Health Study (GS:SFHS) [59], a family-based cohort comprising volunteers from across Scotland, were used to assess two DNAme-based ageing clocks. Finally, the UK Biobank [60] (UKB) was used to test the Clinomics and DEXA clocks trained in ORCADES.

\section{Omics assays}

\section{Dual X-ray absorptiometry (DEXA)}

Whole body imaging was performed on the Hologic fan beam DEXA scanner (GE Healthcare). Measures of body composition were derived from the DEXA scans using APEX2 software for bone, lean and fat tissue and APEX4 software for android, gynoid, visceral and lean fat mass content. 28 measures in the following broad categories: bone mineral density, bone mineral content, fat or lean mass percentages for head, trunk and limbs were selected for analyses. These were measures that did not use chronAge in their calculation and were also available in the UK Biobank. Measures were removed as outliers based on a z-score cut-off of 6 then pre-corrected for sex. Residuals were additionally subject to a threshold by removing outliers with a z-score cut-off of 3 .

\section{DNA methylation}

The Illumina EPIC $850 \mathrm{~K}$ array was used to measure DNA methylation levels in ORCADES. Quality control was carried out using the meffilQC pipeline [61] and minfi package [62]. Samples were excluded as outliers if $>1 \%$ of probes had a detection p-value $>$ 0.01 , due to failure of sex concordance, if samples showed evidence of dye bias or failed median methylation signal z-score cut-off of 3. Probes were removed as outliers if the detection $p$-value was $>0.01$ in $>1 \%$ of samples or had a bead count of $<3$ in at least $5 \%$ of samples. The preprocessNoob function in the "minfi" package was used for array normalisation to remove unwanted technical variation. $M$ values were corrected for the technical covariates: plate number (as a random effect), season of venepuncture, year of venepuncture, plate position and 10 principal components of the control probes (as fixed effects) using GCTA-REML [63].

Instead of creating novel DNA methylation clocks when there are landmark clocks available in the literature, we constructed clocks based on Hannum and Horvath's original epigenetic clocks, to compare with our other omics. As ORCADES used the Illumina EPIC 850k chip rather than the earlier 450k/27k chips used by Hannum and Horvath, our methylation clocks are subsets of Hannum and Horvath's clocks. It has been shown that imputing probes that are absent from the $850 \mathrm{k}$ chip but present in the $450 \mathrm{k} / 27 \mathrm{k}$ set leads to underestimation of both published ageing measures [64]. Thus, for our clocks named Hannum $\mathrm{CpGs}$ and Horvath $\mathrm{CpGs}$ we presented $62 / 71$ and $333 / 353$ of sites, respectively, that were present on the $850 \mathrm{k}$ chip to the penalised regression algorithm for model selection. Residuals from REML within a z-score threshold of 6 were then corrected for sex. 


\section{NMR metabolomics}

The high throughput NMR metabolomics assay of EDTA plasma (Nightingale Health Ltd., Helsinki, Finland) quantified 225 metabolomics measures in molar concentration units. The measures include amino acids, ketone bodies, low molecular weight metabolites and numerous lipid and lipoproteins subclasses. In both ORCADES and Croatia-Korčula, metabolite measures were removed as outliers based on a z-score cut-off of 6 , pre-corrected for sex and the use of statins as a binary variable. Residuals were additionally removed as outliers with a z-score cut-off of 3.

\section{MS fatty acids lipidomics}

Shotgun lipidomics and liquid chromatography tandem mass spectrometry (LC-MS/MS) was used to quantify the molar concentrations of 44 fatty acids as described previously [65]. Fatty acid measures were removed as outliers based on a z-score cut-off of 6 , pre-corrected for sex, box number, plate position and use of statins.

\section{UPLC IgG glycomics}

The glycan data have previously been described in detail by Kristic et al., for the ORCADES [17], CroatiaVis and Croatia-Korcula [56, 57] studies. Raw glycan measures were total area normalised and batch corrected using the "ComBat" function of the sva package [66] in $\mathrm{R}$. The normalised glycan measures were excluded as outliers based on a z-score threshold of 6 and precorrected for sex.

\section{PEA proteomics}

1,102 proteins were measured using a proximity extension assay method (Olink Bioscience, Uppsala, Sweden) [67] from EDTA plasma in 12 x 92-protein panels designated by the manufacturer: cardiovascular 2, cardiovascular 3, inflammation 1, metabolism, cardiometabolic, cell regulation, development, immune response, organ damage, oncology 2, neurology and neuro-exploratory. Measures for all twelve panels are available for 1,057 individuals in ORCADES, with subsets available in Croatia-Vis (inflammation 1, cardiovascular 2 and cardiovascular 3) and EBB (inflammation 1, cardiovascular 2, cardiovascular 3 and oncology 2). PEA proteomicsbased OCAs were re-derived using these subsets to allow comparison across populations. NPX values of proteins (on the $\log 2$ scale) including those nonmissing below the lower limit of detection (LOD), were removed as outliers with a z-score cut-off of 6 . These measures were then pre-corrected for the following covariates via fixed effects linear regression: sex, season of venepuncture, time the plasma sample was in storage between collection and assay (days), plate number, plate row and plate column.

\section{Clinomics}

This dataset consisted of 13 selected clinical measures that are routinely measured during visits with general practitioners and clinicians: albumin, fasting plasma glucose, calcium, uric acid, high density lipoprotein cholesterol, total cholesterol, triglycerides, height, weight, forced expiratory volume in 1 second (FEV1), and diastolic and systolic blood pressure.

\section{MS metabolomics and MS complex lipidomics}

Non-targeted metabolomic and lipidomic features were detected and quantified using Metabolon as described previously [68]. The HD4 dataset comprised measures of 1143 biochemicals while the complex lipids dataset measured 1052 biochemicals, these were treated as two separate omics assays referred to as MS Metabolomics and MS Complex Lipidomics respectively. Measures were removed as outliers with a z-score cut-off of 6 . These measures were then pre-corrected for the following covariates via fixed effects linear regression: sex, statin use, assay run day, plate number and plate row and plate column.

\section{EHR}

The ORCADES cohort has record linkage to hospital admission records (Scottish Morbidity Records: SMR01). The first occurrence of any hospital admission with ICD10 diagnosis, was taken as incidence. NHS Scotland records moved from ICD9 to ICD10 in April 1996, so diagnoses since $\sim 12$ years prior to assessment were captured. The disease groupings analysed included each ICD10 block within 5 Chapters thought a priori to associate with age II (Neoplasms - codes C), IV (Endocrine, nutritional and metabolic diseases - codes E), IX (Diseases of the circulatory system - codes I), and X (Diseases of the respiratory system - codes J). For Chapter II only C codes (malignant) were included in our analyses. Chapters as a whole were also analysed, as were all the diseases from these chapters simultaneously. Incident disease was defined as the time of first hospital admission with a diagnostic code recorded (in any position in the admission record) for any disease within the grouping being analysed. For each disease grouping, subjects with recorded admission prior to the date of venepuncture were then excluded entirely in the subsequent analysis, as already prevalent.

\section{Quality control of omics measures}

Outliers were defined based on z-score thresholds that varied between omics datasets depending on the distributions of the raw measures. Omics measures were pre-corrected for known batch effects and covariates (specified above) using fixed effects linear regression or other specified methods. A second pass z-score threshold on the residuals was used to detect further outliers for a 
subset of assays and all missing values were removed. The residuals produced from covariate correction were then scaled and centred to have a mean of zero and a standard deviation of one to ensure that effect sizes of any variables included in the models were comparable.

\section{Clock construction}

\section{Per omics assay}

The individuals in the ORCADES cohort were split into $75 \%$ training, 25\% testing. For the analysis comparing clock performance across omics platforms the testing $25 \%$ of samples were taken preferentially from the pool of individuals that possess measures for all of the omics platforms. Tenfold cross validation in the training sample was used to select the shrinkage parameter, $\lambda$, for the penalised regression that was estimated to produce the model with the minimum mean squared error. Models were constructed using three different procedures implemented using the glmnet [69] and caret packages in $\mathrm{R}$ with chronAge at venepuncture as the dependent variable: i) least absolute shrinkage and selection operator (LASSO) regression ii) elastic net regression with an alpha of 0.5 iii) elastic net regression with alpha select using 10-fold cross validation in the training sample. We found no difference in performance between the three methods so construction using elastic net regression with an alpha of 0.5 was used throughout the analyses presented. This model was then used to estimate chronAge in the testing sample and an independent out of cohort sample if available.

As stochasticity is present in the procedure, the variables selected for model inclusion will vary depending on the individuals selected to be in the training sample, clock construction was repeated 500 times and the features selected for inclusion and the correlation between chronAge and age estimated by the model were recorded to ensure that the model performance results presented here are representative and not an outlier due to individuals at extreme ends of distributions contributing to the training sample and rare model being used to draw conclusions (data not shown).

\section{Mega-omics}

This model that was presented with all of the features from all of the omics platforms. The dataset itself was created by merging all of the corrected omics measures (residuals) after platform level quality control, again standardising all features to have a mean of zero and standard deviation of one. The clock was created using the same construction procedure outlined above.

\section{Core models}

These models were constructed per omics assay. The elastic net regression algorithm was presented with only those predictors that were selected for model inclusion in $>95 \%$ of the 500 iterations of clock construction for the relevant omics platform. This reduced set of predictors then underwent clock construction as described above.

\section{Principal component clocks}

To ensure that the differences in variance explained in chronAge by different omics clocks is not due to the discrepancy between the number of features available and hence the number of features selected for model inclusion across omics types but rather a genuine difference in the information about ageing captured by different omics; clocks were built using principal components (PCs) of the relevant omics platform as features. The first 3, 5, 10 and 20 PCs were extracted from the covariate corrected scaled and centred omics data at the platform level using the prcomp function in R. These PCs were then presented to the elastic net algorithm and clocks built.

\section{Published clocks}

To compare clocks trained in ORCADES to existing clocks in the literature we calculated four published ageing clocks trained on chronAge: the DNA methylation clock described by Hannum et al., (Hannum 2013) [5], Horvath's DNA methylation-based pan tissue clock (Horvath 2013) [6], the UPLC IgG Glycomics clock GlycanAge [17] and the NMR Metabolomics clock MetaboAge [16]. Hannum 2013 and Horvath 2013 OCAAs were calculated using the online calculator http://dnamage.genetics.ucla.edu/, MetaboAge was calculated using the online calculator metaboage.researchlumc.nl. GlycanAge was calculated as the residuals from the regression of GP6, GP6 ${ }^{2}$, GP14 and GP15 separately for each sex on chronological age at venepuncture.

\section{Correlation of OCAAs}

Pairwise Pearson correlations between 10 of our OCAAs were calculated, Mega-omics OCAA was excluded from this and all between clock comparisons as it contains predictors spanning multiple assays.

\section{Partitioning variance explained in chronAge}

The unique variance in chronAge explained by each clock, $s r_{i}^{2}$, was calculated as the squared part correlation of chronAge $(Y)$ and age estimated by clock $i$ while controlling for all of the other $k$ clocks. Part correlations were calculated using the spcor.test function in the "ppcor" package in $\mathrm{R}$ [70]. The portion of variance in chronAge explained by all of the $k$ clocks together, the $R^{2}$ from the following model:

$$
Y=b_{0}+b_{1} X_{1}+b_{2} X_{2}+\ldots b_{k} X_{k}
$$


Where $Y$ is chronAge and $X_{1 \ldots k}$ are age estimated by clocks 1 to $k$, was used to partition the total variance of chronAge further into that which remains unexplained by the 10 clocks $\left(1-R^{2}\right)$ and that which is explained by overlapping clocks:

$$
1-\left(1-R^{2}\right)-\sum_{i=1}^{k} s r_{i}^{2}
$$

To gain a more detailed insight into the relationship between clocks we carried out pairwise comparisons. Following the same procedure as outlined above, the unique variance in chronAge explained by each clock in the pair is the squared part correlation of chronAge and age estimated by one clock while controlling for age estimated by the other clock in the pair. The variance remaining unexplained by either of the clocks was $1-R^{2}$ of a bivariate model. The overlap, calculated by subtraction, is specifically the variance in chronAge explained by both of the clocks in the pair. This is unlike overlap calculated in the previous step, where we were only able to state that this variance was not unique to a particular clock but unable to deconstruct further.

\section{Assessing the overlap between clocks}

We assessed whether the combined variance in chronAge explained by pairs of clocks deviated from what would be expected by chance if both clocks were independently sampling from a latent set of predictors (ISLSP) of chronAge. The combined variance in chronAge explained by both clocks together was calculated as the multiple $R^{2}$ from a bivariate model, with chronAge being the dependent variable and the estimated ages from the two clocks in the pair the independent variables. The variance explained in chronAge $\left(v_{i}\right)$ by each clock $(i)$ individually was the univariate $R^{2}$ from the regression of estimated age on chronAge. The expected variance in chronAge explained by two clocks by chance $(E)$ was calculated as follows:

$$
E=1-\left(1-v_{1}\right)\left(1-v_{2}\right)
$$

The idea being that the variance in chronAge not already explained by the first clock is $1-v_{i}$. With the null hypothesis that the two clocks are independent samples from the latent set of complete predictors and thus explain partly overlapping information about age. The expected left unexplained after the addition of the second clock is thus $\left(1-v_{1}\right)\left(1-v_{2}\right)$.

To allow for the comparison of the deviation of observed variance explained in chronAge $(O)$ from expected $(E)$ across pairs of clocks, this deviation was re-scaled. As the magnitude of $v_{i}$ effects the possible range of values $O$ could take. The theoretical minimum variance explained $\left(E_{\min }\right)$ by two clocks is the variance explained by the larger of the two clocks alone (the second clock only providing information already captured by the first). The theoretical maximum $\left(E_{\max }\right)$ is $v_{1}+v_{2}$ or 1 if $v_{1}+v_{2}>1$ (the clocks are explaining entirely non-overlapping variance). Comparisons containing clocks with high $v_{i}$ will have a much smaller range of possible $O$ than those with low $v_{i}$ so directly comparing the magnitude of the deviation of observed from expected is not ideal. The results presented are on a scale of excess overlap calculated as follows:

$$
\frac{E-O}{E-E_{\min }}
$$

With a value of 0 meaning that the observed variance explained equals that expected by chance if the clocks were independent. A value of 1 denoting that no additional variance was explained with the addition of the second clock. Negative values mean that the two clocks overlap less than expected and track separate aspects of chronological ageing.

\section{Association with health-related phenotypes and incident disease}

OCAAs were tested for association with health-related risk factors and age-related incident diseases, as measured by hospital admission.

\section{Association with chronAge}

We first tested whether the risk factors and disease outcomes were associated with chronAge. For incident disease: time from assessment to incidence or to study end (the date when SMR01 records were extracted: December 2017, around ten years after assessment) was modelled using a Cox proportional hazard model [71] and the Surv function in the "survival" package in R. Subjects with prevalent disease were excluded. The baseline hazard was dependent on time since assessment, and hazards ratios dependent on chronAge and sex. We used time since assessment as the determinant of base hazard rather than chronAge, so that we could determine which groupings had stronger age-related effects and compare the effects of OCAA to those of chronAge. P-values for association with chronAge (and later OCAA) were calculated using a one-sided test, with $\mathrm{H} 1$ being that chronAge (or OCAA) increased risk.

\section{Association with OCAAs}

with standardised risk factors (units of phenotypic standard deviation) were carried out using linear regression with chronAge and sex fitted as fixed effects 
covariates. To restrict the burden of multiple testing we only tested the association of OCAAs on risk factors or disease blocks which showed a statistically significant association (effect size $>0$ ) with chronAge at outset (Benjamini-Hochberg FDR $<10 \%$ ) and had $>5$ incident cases (disease blocks). We tested the effect of OCAAs on each disease grouping using the same model as for chronAge, including chronAge and OCAA as effects. OCAA was not standardised but observed effect sizes were rescaled (divided) by the effect of chronAge, using the same model, enabling a comparison of the effect of one year's OCAA with one year's chronAge, with a value of 1 denoting the same effect. False discovery rate was again determined using the Benjamini-Hochberg method $($ FDR $<10 \%)$.

Across both risk factors and disease, we found that large estimated effects arose in the context of large SEs. To facilitate visualising the results we had most confidence in we applied a shrinkage method, imposing a prior assumption on the distribution of beta (mean 0, SD 1) to the likelihood of our observed beta, shrinking resultant estimates with larger SEs more towards 0.

Individual tests of association generally had limited power due to multiple testing and the low variance of OCAA (compared with chronAge). We therefore considered the results of each OCAA across multiple outcomes by inverse variance weighting (IVW) observed results for individual outcomes. The covariance amongst outcomes and predictors, mean that the independence assumption for meta-analysis (or sign testing) is violated. Whilst this should not bias estimates, their precision will be overstated. We consider these results to be descriptive, and not conformable to formal testing. We use " $\sim$ " to denote SEs calculated under the violated independence assumption, but still consider these useful to give a sense of magnitude. Conversely, for the same reason, the formal tests we perform (FDRs) are likely to be conservative.

We repeated these analyses with standardised OCAAs to compare the prognostic ability of different OCAAs at a population level, across risk factors and diseases and with our PC clocks OCAAs.

\section{Data availability}

There is neither research ethics committee approval, nor consent from individual participants, to permit open release of the individual level research data underlying this study. Please contact the QTL Data Access Committee (accessQTL@ed.ac.uk) for further information if required. Access to data from GS:SFHS is by application (access@generationscotland.org) and a managed process. Access to data from the Estonian
Biobank is by request and managed by the Estonian Committee on Bioethics and Human Research.

\section{AUTHOR CONTRIBUTIONS}

E.M.D: Project conception, methodology, manuscript drafting and editing, formal analysis, visualisation. N.T: Performed validation analysis in Estonian Biobank. L.K: Processed and performed QC on the UPLC IgG Glycomics data in ORCADES, Croatia-Vis and CroatiaKorcula and supervised A.F. A.F: Processed and performed QC on the DNA methylation data in ORCADES. R.W: Preparation of the DNA methylation data in GS:SFHS. P.K.J: Project conception, methodology, formal analysis, manuscript drafting and editing, project supervision. J.F.W: Project conception, manuscript drafting and editing, project supervision. All other authors commented and approved the manuscript prior to submission.

\section{ACKNOWLEDGMENTS AND FUNDING}

We thank Archie Campbell for assistance with the electronic health records from ORCADES. The Orkney Complex Disease Study (ORCADES) was supported by the Chief Scientist Office of the Scottish Government (CZB/4/276, CZB/4/710), a Royal Society URF to J.F.W., the MRC Human Genetics Unit quinquennial programme "QTL in Health and Disease", Arthritis Research UK and the European Union framework program 6 EUROSPAN project (contract no. LSHGCT-2006-018947). DNA extractions were performed at the Edinburgh Clinical Research Facility, University of Edinburgh. We would like to acknowledge the invaluable contributions of the research nurses in Orkney, the administrative team in Edinburgh and the people of Orkney. We acknowledge support from the MRC Human Genetics Unit programme grant, "Quantitative traits in health and disease" (U. MC_UU_00007/10).

The CROATIA-Vis and, CROATIA-Korcula studies were funded by grants from the Medical Research Council (UK), from the Republic of Croatia Ministry of Science, Education and Sports (108-1080315-0302; 2161080315-0302) and the Croatian Science Foundation (8875); and the CROATIA-Korčula genotyping was funded by the European Union framework program 6 project EUROSPAN (LSHGCT2006018947). We thank the staff of several institutions in Croatia that supported the field work, including Zagreb Medical Schools, the Institute for Anthropological Research in Zagreb, the recruitment team from the Croatian Centre for Global Health, University of Split and all the study participants. We are grateful to the Helmholtz Zentrum Munchen (Munich, Germany), AROS Applied Biotechnology, 
(Aarhus, Denmark) and the Edinburgh Clinical Research facility, University of Edinburgh (Edinburgh, United Kingdom) for SNP array genotyping.

Generation Scotland received core support from the Chief Scientist Office of the Scottish Government Health Directorates [CZD/16/6] and the Scottish Funding Council [HR03006] and is currently supported by the Wellcome Trust [216767/Z/19/Z]. Genotyping of the GS:SFHS samples was carried out by the Genetics Core Laboratory at the Edinburgh Clinical Research Facility, University of Edinburgh, Scotland and was funded by the Medical Research Council UK and the Wellcome Trust (Wellcome Trust Strategic Award "STratifying Resilience and Depression Longitudinally" (STRADL) Reference 104036/Z/14/Z). We are grateful to all the families who took part, the general practitioners and the Scottish School of Primary Care for their help in recruiting them, and the whole Generation Scotland team, which includes interviewers, computer and laboratory technicians, clerical workers, research scientists, volunteers, managers, receptionists, healthcare assistants and nurses. Ethical approval for the GS:SFHS study was obtained from the Tayside Committee on Medical Research Ethics (on behalf of the National Health Service).

This research was funded by the European Union through the European Regional Development Fund (N.T.) and SP1GI18045T (T.E., N.T.), grants PRG1291 (T.E., N.T.) and PRG 1197 (K.F., N.T.) of the Estonian Research Council. The Estonian Biobank study was approved by the Ethics Review Committee on Human Research of the University of Tartu and the Estonian Genome Center, University of Tartu scientific committee. Written informed consent was obtained from participants in accordance with the Declaration of Helsinki. The work of L.K. was supported by an RCUK Innovation Fellowship from the National Productivity Investment Fund (MR/R026408/1).

We thank the UK Biobank Resource, approved under application 19655. We acknowledge funding from the UK Medical Research Council Doctoral Training Programme in Precision Medicine (MR/N013166/1).

\section{CONFLICTS OF INTEREST}

P.K.J is a paid consultant for Humanity Inc. and Global Gene Corporation.

\section{REFERENCES}

1. López-Otín C, Blasco MA, Partridge $L$, Serrano $M$, Kroemer G. The hallmarks of aging. Cell. 2013; 153:1194-217. https://doi.org/10.1016/i.cell.2013.05.039 PMID:23746838

2. Levine ME, Lu AT, Quach $A$, Chen BH, Assimes TL, Bandinelli S, Hou L, Baccarelli AA, Stewart JD, Li Y, Whitsel EA, Wilson JG, Reiner AP, et al. An epigenetic biomarker of aging for lifespan and healthspan. Aging (Albany NY). 2018; 10:573-91. https://doi.org/10.18632/aging.101414 PMID:29676998

3. Horvath $S$, Singh K, Raj K, Khairnar S, Sanghavi A, Shrivastava A, Zoller JA, Li CZ, Herenu CB, CanatelliMallat M, Lehmann M, Solberg Woods LC, Garcia Martinez A, et al. Reversing age: dual species measurement of epigenetic age with a single clock. bioRxiv. 2020. [Epub ahead of print]. https://doi.org/10.1101/2020.05. $\underline{07.082917}$

4. Baker GT 3rd, Sprott RL. Biomarkers of aging. Exp Gerontol. 1988; 23:223-39. https://doi.org/10.1016/0531-5565(88)90025-3 PMID: $\underline{3058488}$

5. Hannum G, Guinney J, Zhao L, Zhang L, Hughes G, Sadda S, Klotzle B, Bibikova M, Fan JB, Gao Y, Deconde $R$, Chen M, Rajapakse I, et al. Genome-wide methylation profiles reveal quantitative views of human aging rates. Mol Cell. 2013; 49:359-67. https://doi.org/10.1016/j.molcel.2012.10.016 PMID:23177740

6. Horvath S. DNA methylation age of human tissues and cell types. Genome Biol. 2013; 14:R115. https://doi.org/10.1186/gb-2013-14-10-r115 PMID:24138928

7. Marioni RE, Shah S, McRae AF, Chen BH, Colicino E, Harris SE, Gibson J, Henders AK, Redmond P, Cox SR, Pattie A, Corley J, Murphy L, et al. DNA methylation age of blood predicts all-cause mortality in later life. Genome Biol. 2015; 16:25. https://doi.org/10.1186/s13059-015-0584-6 PMID:25633388

8. Bocklandt S, Lin W, Sehl ME, Sánchez FJ, Sinsheimer JS, Horvath S, Vilain E. Epigenetic predictor of age. PLoS One. 2011; 6:e14821. https://doi.org/10.1371/journal.pone.0014821 PMID:21731603

9. Jansen R, Han LK, Verhoeven JE, Aberg KA, van den Oord EC, Milaneschi Y, Penninx BW. An integrative study of five biological clocks in somatic and mental health. Elife. 2021; 10:e59479.

https://doi.org/10.7554/eLife.59479

PMID:33558008

10. Zhang WG, Zhu SY, Bai XJ, Zhao DL, Jian SM, Li J, Li ZX, 
Fu B, Cai GY, Sun XF, Chen XM. Select aging biomarkers based on telomere length and chronological age to build a biological age equation. Age (Dordr). 2014; 36:9639.

https://doi.org/10.1007/s11357-014-9639-y

PMID:24659482

11. Chen W, Qian W, Wu G, Chen W, Xian B, Chen X, Cao Y, Green CD, Zhao F, Tang K, Han JD. Three-dimensional human facial morphologies as robust aging markers. Cell Res. 2015; 25:574-87.

https://doi.org/10.1038/cr.2015.36

PMID:25828530

12. Cole JH, Poudel RP, Tsagkrasoulis D, Caan MW, Steves C, Spector TD, Montana G. Predicting brain age with deep learning from raw imaging data results in a reliable and heritable biomarker. Neuroimage. 2017; 163:115-24.

https://doi.org/10.1016/i.neuroimage.2017.07.059 PMID:28765056

13. Cole JH, Ritchie SJ, Bastin ME, Valdés Hernández MC, Muñoz Maniega S, Royle N, Corley J, Pattie A, Harris $\mathrm{SE}$, Zhang $\mathrm{Q}$, Wray NR, Redmond $\mathrm{P}$, Marioni RE, et al. Brain age predicts mortality. Mol Psychiatry. 2018; 23:1385-92.

https://doi.org/10.1038/mp.2017.62 PMID:28439103

14. Goyal MS, Blazey TM, Su Y, Couture LE, Durbin TJ, Bateman RJ, Benzinger TL, Morris JC, Raichle ME, Vlassenko AG. Persistent metabolic youth in the aging female brain. Proc Natl Acad Sci USA. 2019; 116:3251-5.

https://doi.org/10.1073/pnas.1815917116

PMID: $\underline{0718410}$

15. Sokolova K, Barker GJ, Montana G. Convolutional neural-network-based ordinal regression for brain age prediction from MRI scans. SPIE Medical Imaging 2020; 11313: 113132B.

https://doi.org/10.1117/12.2549636

16. van den Akker EB, Trompet S, Barkey Wolf JJ, Beekman $M$, Suchiman HE, Deelen J, Asselbergs FW, Boersma E, Cats D, Elders PM, Geleijnse JM, Ikram MA, Kloppenburg $M$, et al. Metabolic Age Based on the BBMRI-NL ${ }^{1} \mathrm{H}-\mathrm{NMR}$ Metabolomics Repository as Biomarker of Age-related Disease. Circ Genom Precis Med. 2020; 13:541-7.

https://doi.org/10.1161/CIRCGEN.119.002610 PMID:33079603

17. Krištić J, Vučković F, Menni C, Klarić L, Keser T, Beceheli I, Pučić-Baković M, Novokmet M, Mangino M, Thaqi K, Rudan P, Novokmet N, Sarac J, et al. Glycans are a novel biomarker of chronological and biological ages. J Gerontol A Biol Sci Med Sci. 2014; 69:779-89.

https://doi.org/10.1093/gerona/glt190 PMID:24325898
18. Enroth S, Enroth SB, Johansson Å, Gyllensten U. Protein profiling reveals consequences of lifestyle choices on predicted biological aging. Sci Rep. 2015; 5:17282.

https://doi.org/10.1038/srep17282 PMID:26619799

19. Lehallier B, Gate D, Schaum N, Nanasi T, Lee SE, Yousef H, Moran Losada P, Berdnik D, Keller A, Verghese J, Sathyan S, Franceschi C, Milman S, et al. Undulating changes in human plasma proteome profiles across the lifespan. Nat Med. 2019; 25:1843-50. https://doi.org/10.1038/s41591-019-0673-2 PMID:31806903

20. Lehallier B, Shokhirev MN, Wyss-Coray T, Johnson AA. Data mining of human plasma proteins generates a multitude of highly predictive aging clocks that reflect different aspects of aging. Aging Cell. 2020; 19:e13256. https://doi.org/10.1111/acel.13256 PMID: $\underline{3031577}$

21. Alpert A, Pickman $Y$, Leipold M, Rosenberg-Hasson Y, Ji $X$, Gaujoux R, Rabani H, Starosvetsky E, Kveler K, Schaffert S, Furman D, Caspi O, Rosenschein U, et al. A clinically meaningful metric of immune age derived from high-dimensional longitudinal monitoring. Nat Med. 2019; 25:487-95.

https://doi.org/10.1038/s41591-019-0381-y PMID: $\underline{30842675}$

22. Lu AT, Quach A, Wilson JG, Reiner AP, Aviv A, Raj K, Hou L, Baccarelli AA, Li Y, Stewart JD, Whitsel EA, Assimes TL, Ferrucci L, Horvath S. DNA methylation GrimAge strongly predicts lifespan and healthspan. Aging (Albany NY). 2019; 11:303-27.

https://doi.org/10.18632/aging.101684 PMID:30669119

23. Belsky DW, Moffitt TE, Cohen AA, Corcoran DL, Levine ME, Prinz JA, Schaefer J, Sugden K, Williams B, Poulton $\mathrm{R}$, Caspi A. Eleven Telomere, Epigenetic Clock, and Biomarker-Composite Quantifications of Biological Aging: Do They Measure the Same Thing? Am J Epidemiol. 2018; 187:1220-30.

https://doi.org/10.1093/aje/kwx346 PMID:29149257

24. Maddock J, Castillo-Fernandez J, Wong A, Cooper R, Richards M, Ong KK, Ploubidis GB, Goodman A, Kuh D, Bell JT, Hardy R. DNA Methylation Age and Physical and Cognitive Aging. J Gerontol A Biol Sci Med Sci. 2020; 75:504-11.

https://doi.org/10.1093/gerona/glz246 PMID:31630156

25. Li X, Ploner A, Wang Y, Magnusson PK, Reynolds C, Finkel D, Pedersen NL, Jylhävä J, Hägg S. Longitudinal trajectories, correlations and mortality associations of nine biological ages across 20-years follow-up. Elife. 2020; 9:e51507.

https://doi.org/10.7554/eLife.51507 
PMID:32041686

26. Hillary RF, Stevenson AJ, McCartney DL, Campbell A, Walker RM, Howard DM, Ritchie CW, Horvath S, Hayward C, McIntosh AM, Porteous DJ, Deary IJ, Evans $\mathrm{KL}$, Marioni RE. Epigenetic measures of ageing predict the prevalence and incidence of leading causes of death and disease burden. Clin Epigenetics. 2020; 12:115.

https://doi.org/10.1186/s13148-020-00905-6 PMID:32736664

27. Zheng $\mathrm{Y}$, Joyce $\mathrm{BT}$, Colicino $\mathrm{E}$, Liu L, Zhang $\mathrm{W}$, Dai $\mathrm{Q}$, Shrubsole MJ, Kibbe WA, Gao T, Zhang Z, Jafari N, Vokonas P, Schwartz J, et al. Blood Epigenetic Age may Predict Cancer Incidence and Mortality. EBioMedicine. 2016; 5:68-73.

https://doi.org/10.1016/i.ebiom.2016.02.008 PMID:27077113

28. Horvath S, Gurven M, Levine ME, Trumble BC, Kaplan $H$, Allayee H, Ritz BR, Chen B, Lu AT, Rickabaugh TM, Jamieson BD, Sun D, Li S, et al. An epigenetic clock analysis of race/ethnicity, sex, and coronary heart disease. Genome Biol. 2016; 17:171.

https://doi.org/10.1186/s13059-016-1030-0

PMID:27511193

29. McQuillan $R$, Leutenegger $A L$, Abdel-Rahman $R$, Franklin CS, Pericic M, Barac-Lauc L, Smolej-Narancic N, Janicijevic B, Polasek O, Tenesa A, Macleod AK, Farrington SM, Rudan $\mathrm{P}$, et al. Runs of homozygosity in European populations. Am J Hum Genet. 2008; 83:359-72.

https://doi.org/10.1016/j.ajhg.2008.08.007 PMID:18760389

30. Franke K, Ziegler G, Klöppel S, Gaser C, and Alzheimer's Disease Neuroimaging Initiative. Estimating the age of healthy subjects from T1-weighted MRI scans using kernel methods: exploring the influence of various parameters. Neuroimage. 2010; 50:883-92. https://doi.org/10.1016/i.neuroimage.2010.01.005 PMID:20070949

31. Zhang $\mathrm{Q}$, Vallerga $\mathrm{CL}$, Walker RM, Lin $\mathrm{T}$, Henders $\mathrm{AK}$, Montgomery GW, He J, Fan D, Fowdar J, Kennedy M, Pitcher T, Pearson J, Halliday G, et al. Improved precision of epigenetic clock estimates across tissues and its implication for biological ageing. Genome Med. 2019; 11:54.

https://doi.org/10.1186/s13073-019-0667-1 PMID:31443728

32. Pyrkov TV, Fedichev PO. Biological age is a universal marker of aging, stress, and frailty. bioRxiv. 2019. [Epub ahead of print]. https://doi.org/10.1101/578245

33. Alsaleh $\mathrm{H}$, Haddrill PR. Identifying blood-specific agerelated DNA methylation markers on the Illumina
MethylationEPIC ${ }^{\circledR}$ BeadChip. Forensic Sci Int. 2019; 303:109944.

https://doi.org/10.1016/j.forsciint.2019.109944 PMID:31546163

34. Horvath S, Pirazzini C, Bacalini MG, Gentilini D, Di Blasio AM, Delledonne M, Mari D, Arosio B, Monti D, Passarino G, De Rango F, D’Aquila P, Giuliani C, et al. Decreased epigenetic age of PBMCs from Italian semisupercentenarians and their offspring. Aging (Albany NY). 2015; 7:1159-70. https://doi.org/10.18632/aging.100861 PMID:26678252

35. Chen BH, Marioni RE, Colicino E, Peters MJ, WardCaviness CK, Tsai PC, Roetker NS, Just AC, Demerath EW, Guan W, Bressler J, Fornage M, Studenski S, et al. DNA methylation-based measures of biological age: meta-analysis predicting time to death. Aging (Albany NY). 2016; 8:1844-65.

https://doi.org/10.18632/aging.101020 PMID:27690265

36. Christiansen L, Lenart A, Tan Q, Vaupel JW, Aviv A, McGue $M$, Christensen K. DNA methylation age is associated with mortality in a longitudinal Danish twin study. Aging Cell. 2016; 15:149-54.

https://doi.org/10.1111/acel.12421

PMID:26594032

37. Perna L, Zhang Y, Mons U, Holleczek B, Saum KU, Brenner $\mathrm{H}$. Epigenetic age acceleration predicts cancer, cardiovascular, and all-cause mortality in a German case cohort. Clin Epigenetics. 2016; 8:64. https://doi.org/10.1186/s13148-016-0228-z PMID:27274774

38. Levine ME, Lu AT, Bennett DA, Horvath S. Epigenetic age of the pre-frontal cortex is associated with neuritic plaques, amyloid load, and Alzheimer's disease related cognitive functioning. Aging (Albany NY). 2015; 7:1198-211.

https://doi.org/10.18632/aging.100864 PMID:26684672

39. Vidal L, Lopez-Golan Y, Rego-Perez I, Horvath S, Blanco FJ, Riancho JA, Gomez-Reino JJ, Gonzalez A. Specific increase of methylation age in osteoarthritis cartilage. Osteoarthritis Cartilage. 2016; 24:S63. https://doi.org/10.1016/i.joca.2016.01.140

40. Marioni RE, Shah S, McRae AF, Ritchie SJ, MunizTerrera G, Harris SE, Gibson J, Redmond P, Cox SR, Pattie A, Corley J, Taylor A, Murphy L, et al. The epigenetic clock is correlated with physical and cognitive fitness in the Lothian Birth Cohort 1936. Int J Epidemiol. 2015; 44:1388-96. https://doi.org/10.1093/ije/dyu277 PMID:25617346

41. Horvath S, Ritz BR. Increased epigenetic age and 
granulocyte counts in the blood of Parkinson's disease patients. Aging (Albany NY). 2015; 7:1130-42.

https://doi.org/10.18632/aging.100859

PMID:26655927

42. Maierhofer A, Flunkert J, Oshima J, Martin GM, Haaf T, Horvath S. Accelerated epigenetic aging in Werner syndrome. Aging (Albany NY). 2017; 9:1143-52.

https://doi.org/10.18632/aging.101217

PMID:28377537

43. Horvath S, Langfelder P, Kwak S, Aaronson J, Rosinski J, Vogt TF, Eszes M, Faull RL, Curtis MA, Waldvogel HJ, Choi OW, Tung S, Vinters HV, et al. Huntington's disease accelerates epigenetic aging of human brain and disrupts DNA methylation levels. Aging (Albany NY). 2016; 8:1485-512.

https://doi.org/10.18632/aging.101005

PMID:27479945

44. Breitling LP, Saum KU, Perna L, Schöttker B, Holleczek $\mathrm{B}$, Brenner $\mathrm{H}$. Frailty is associated with the epigenetic clock but not with telomere length in a German cohort. Clin Epigenetics. 2016; 8:21.

https://doi.org/10.1186/s13148-016-0186-5

PMID:26925173

45. Walker RF, Liu JS, Peters BA, Ritz BR, Wu T, Ophoff RA, Horvath $S$. Epigenetic age analysis of children who seem to evade aging. Aging (Albany NY). 2015; 7:334-9.

https://doi.org/10.18632/aging.100744

PMID:25991677

46. Horvath S, Levine AJ. HIV-1 Infection Accelerates Age According to the Epigenetic Clock. J Infect Dis. 2015; 212:1563-73.

https://doi.org/10.1093/infdis/jiv277

PMID:25969563

47. Levine ME, Lu AT, Chen BH, Hernandez DG, Singleton $A B$, Ferrucci L, Bandinelli S, Salfati E, Manson JE, Quach $A$, Kusters CD, Kuh D, Wong $A$, et al. Menopause accelerates biological aging. Proc Natl Acad Sci USA. 2016; 113:9327-32.

https://doi.org/10.1073/pnas.1604558113

PMID:27457926

48. Singh SS, Heijmans R, Meulen CK, Lieverse AG, Gornik $\mathrm{O}$, Sijbrands EJ, Lauc G, van Hoek M. Association of the IgG N-glycome with the course of kidney function in type 2 diabetes. BMJ Open Diabetes Res Care. 2020; 8:e001026.

https://doi.org/10.1136/bmjdrc-2019-001026

PMID:32349995

49. Lemmers RF, Vilaj $M$, Urda D, Agakov F, Šimurina $M$, Klaric L, Rudan I, Campbell H, Hayward C, Wilson JF, Lieverse AG, Gornik O, Sijbrands EJ, et al. IgG glycan patterns are associated with type 2 diabetes in independent European populations. Biochim Biophys
Acta Gen Subj. 2017; 1861:2240-9.

https://doi.org/10.1016/i.bbagen.2017.06.020 PMID:28668296

50. Menni C, Gudelj I, Macdonald-Dunlop E, Mangino M, Zierer J, Bešić E, Joshi PK, Trbojević-Akmačić I, Chowienczyk PJ, Spector TD, Wilson JF, Lauc G, Valdes AM. Glycosylation Profile of Immunoglobulin G Is Cross-Sectionally Associated With Cardiovascular Disease Risk Score and Subclinical Atherosclerosis in Two Independent Cohorts. Circ Res. 2018; 122:1555-64.

https://doi.org/10.1161/CIRCRESAHA.117.312174 PMID:29535164

51. Liu Z, Leung D, Levine M. Comparative analysis of epigenetic aging clocks from CpG characteristics to functional associations. bioRxiv. 2019. [Epub ahead of print]. https://doi.org/10.1101/512483

52. Lee $Y$, Haftorn KL, Denault WR, Nustad HE, Page CM, Lyle R, Lee- $\varnothing$ degård $S$, Moen GH, Prasad RB, Groop LC, Sletner L, Sommer C, Magnus MC, et al. Bloodbased epigenetic estimators of chronological age in human adults using DNA methylation data from the Illumina MethylationEPIC array. BMC Genomics. 2020; 21:747.

https://doi.org/10.1186/s12864-020-07168-8

PMID:33109080

53. Zenin A, Tsepilov Y, Sharapov S, Getmantsev E, Menshikov LI, Fedichev PO, Aulchenko Y. Identification of 12 genetic loci associated with human healthspan. Commun Biol. 2019; 2:41.

https://doi.org/10.1038/s42003-019-0290-0 PMID:30729179

54. McCrory C, Fiorito G, Hernandez B, Polidoro S, O'Halloran AM, Hever A, Ni Cheallaigh C, Lu AT, Horvath S, Vineis P, Kenny RA. GrimAge Outperforms Other Epigenetic Clocks in the Prediction of AgeRelated Clinical Phenotypes and All-Cause Mortality. J Gerontol A Biol Sci Med Sci. 2021; 76:741-9.

https://doi.org/10.1093/gerona/glaa286 PMID:33211845

55. Deelen J, Kettunen J, Fischer K, van der Spek A, Trompet S, Kastenmüller G, Boyd A, Zierer J, van den Akker EB, Ala-Korpela M, Amin N, Demirkan A, Ghanbari $M$, et al. A metabolic profile of all-cause mortality risk identified in an observational study of 44,168 individuals. Nat Commun. 2019; 10:3346.

https://doi.org/10.1038/s41467-019-11311-9 PMID:31431621

56. Pucić M, Knezević A, Vidic J, Adamczyk B, Novokmet M, Polasek O, Gornik O, Supraha-Goreta S, Wormald MR, Redzić I, Campbell H, Wright A, Hastie ND, et al. High throughput isolation and glycosylation analysis of IgGvariability and heritability of the IgG glycome in three 
isolated human populations. Mol Cell Proteomics. 2011; 10:M111.010090.

https://doi.org/10.1074/mcp.M111.010090

PMID:21653738

57. Klarić L, Tsepilov YA, Stanton CM, Mangino M, Sikka TT, Esko T, Pakhomov E, Salo P, Deelen J, McGurnaghan SJ, Keser T, Vučković F, Ugrina I, et al. Glycosylation of immunoglobulin $\mathrm{G}$ is regulated by a large network of genes pleiotropic with inflammatory diseases. Sci Adv. 2020; 6:eaax0301.

https://doi.org/10.1126/sciadv.aax0301

PMID:32128391

58. Leitsalu L, Haller T, Esko T, Tammesoo ML, Alavere $H$, Snieder H, Perola M, Ng PC, Mägi R, Milani L, Fischer K, Metspalu A. Cohort Profile: Estonian Biobank of the Estonian Genome Center, University of Tartu. Int J Epidemiol. 2015; 44:1137-47.

https://doi.org/10.1093/ije/dyt268 PMID:24518929

59. Smith BH, Campbell A, Linksted $P$, Fitzpatrick B, Jackson C, Kerr SM, Deary IJ, Macintyre DJ, Campbell H, McGilchrist M, Hocking LJ, Wisely L, Ford I, et al. Cohort Profile: Generation Scotland: Scottish Family Health Study (GS:SFHS). The study, its participants and their potential for genetic research on health and illness. Int J Epidemiol. 2013; 42:689-700.

https://doi.org/10.1093/ije/dys084

PMID:22786799

60. Sudlow C, Gallacher J, Allen N, Beral V, Burton P, Danesh J, Downey P, Elliott P, Green J, Landray M, Liu $B$, Matthews $P$, Ong $G$, et al. UK biobank: an open access resource for identifying the causes of a wide range of complex diseases of middle and old age. PLoS Med. 2015; 12:e1001779.

https://doi.org/10.1371/journal.pmed.1001779

PMID:25826379

61. Min JL, Hemani G, Davey Smith G, Relton C, Suderman M. Meffil: efficient normalization and analysis of very large DNA methylation datasets. Bioinformatics. 2018; 34:3983-9.

https://doi.org/10.1093/bioinformatics/bty476 PMID:29931280

62. Aryee MJ, Jaffe AE, Corrada-Bravo H, Ladd-Acosta C, Feinberg AP, Hansen KD, Irizarry RA. Minfi: a flexible and comprehensive Bioconductor package for the analysis of Infinium DNA methylation microarrays. Bioinformatics. 2014; 30:1363-9.

https://doi.org/10.1093/bioinformatics/btu049 PMID:24478339

63. Yang J, Lee SH, Goddard ME, Visscher PM. GCTA: a tool for genome-wide complex trait analysis. Am J Hum Genet. 2011; 88:76-82. https://doi.org/10.1016/j.ajhg.2010.11.011 PMID:21167468

64. Dhingra R, Kwee LC, Diaz-Sanchez D, Devlin RB, Cascio W, Hauser ER, Gregory S, Shah S, Kraus WE, Olden K, Ward-Caviness CK. Evaluating DNA methylation age on the Illumina MethylationEPIC Bead Chip. PLoS One. 2019; 14:e0207834. https://doi.org/10.1371/journal.pone.0207834 PMID:31002714

65. Sales S, Graessler J, Ciucci S, Al-Atrib R, Vihervaara T, Schuhmann K, Kauhanen D, Sysi-Aho M, Bornstein SR, Bickle M, Cannistraci CV, Ekroos K, Shevchenko A. Gender, Contraceptives and Individual Metabolic Predisposition Shape a Healthy Plasma Lipidome. Sci Rep. 2016; 6:27710.

https://doi.org/10.1038/srep27710

PMID:27295977

66. Leek JT. svaseq: removing batch effects and other unwanted noise from sequencing data. Nucleic Acids Res. 2014; 42:e161. https://doi.org/10.1093/nar/gku864 PMID:25294822

67. Lundberg $M$, Eriksson A, Tran B, Assarsson E, Fredriksson S. Homogeneous antibody-based proximity extension assays provide sensitive and specific detection of low-abundant proteins in human blood. Nucleic Acids Res. 2011; 39:e102.

https://doi.org/10.1093/nar/gkr424 PMID:21646338

68. Long T, Hicks M, Yu HC, Biggs WH, Kirkness EF, Menni C, Zierer J, Small KS, Mangino M, Messier H, Brewerton $S$, Turpaz Y, Perkins BA, et al. Whole-genome sequencing identifies common-to-rare variants associated with human blood metabolites. Nat Genet. 2017; 49:568-78.

https://doi.org/10.1038/ng.3809

PMID:28263315

69. Friedman J, Hastie T, Tibshirani R. Regularization Paths for Generalized Linear Models via Coordinate Descent. J Stat Softw. 2010; 33:1-22. https://doi.org/10.18637/jss.v033.i01 PMID:20808728

70. Kim S. ppcor: An R Package for a Fast Calculation to Semi-partial Correlation Coefficients. Commun Stat Appl Methods. 2015; 22:665-74. https://doi.org/10.5351/CSAM.2015.22.6.665 PMID:26688802

71. Cox DR. Regression Models and Life-Tables. J R Stat Soc B. 1972; 34:187-202. https://doi.org/10.1111/i.2517-6161.1972.tb00899.x 


\title{
SUPPLEMENTARY MATERIALS
}

\author{
Supplementary Note
}

\section{Replication of omics ageing clocks trained in ORCADES in independent populations}

We found clocks built using the subsets of PEA proteomics measures available in our validation cohorts correlating with chronAge nearly as highly in CroatiaVis $(r=0.89)$ and EBB $(r=0.91)$ as in the ORCADES testing sample ( $\mathrm{r}=0.91$ and $\mathrm{r}=0.93)$ (Supplementary Figure 3). Similarly, both of our own DNAme Hannum and Horvath $\mathrm{CpG}$ based clocks achieved comparable correlations between OCA and chronAge in EBB (Hannum: $\mathrm{r}=0.98$, Horvath: $\mathrm{r}=0.97$ ) and GS:SHFS (DNAme Hannum CpGs: r=0.96, DNAme Horvath CpGs: $r=0.93$ ) as in the ORCADES testing sample (DNAme Hannum CpGs: r=0.96, DNAme Horvath CpGs: $r=0.93$ ). Our UPLC IgG glycomics and Clinomics OCA were still correlated with chronAge in independent cohorts (UPLC IgG glycomics: $\mathrm{r}=0.62$ Croatia-Vis, $\mathrm{r}=0.61$ Croatia-Korcula, Clinomics: $\mathrm{r}=0.56 \mathrm{UKBB}$ ) but less than in the ORCADES testing sample (UPLC IgG glycomics: $r=0.74$, Clinomics: $r=0.80$ ). There was correlation between NMR metabolomics estimated age and chronAge in Croatia-Korcula, $\mathrm{r}=0.55$ compared to $\mathrm{r}=0.73$ in ORCADES however only a correlation of $r=0.26$ in EBB. Similarly, we found that the DEXA estimated age in UKBB correlated substantially lower with chronAge than in ORCADES (UKBB: $r=0.30$, ORCADES: $r=0.66$ ).

To assess whether the poor correlation of DEXA OCA and chronAge in UKBB was due to the difference in the ranges of chronAge of individuals in ORCADES compared to the UKBB we also compared a clock that was evaluated in ORCADES individuals between 40-75 (the recruiting age range of UKBB, compared to the 16100 in the full ORCADES dataset). Despite the DEXA OCA having a lower correlation with chronAge in the age restricted ORCADES sample, $\mathrm{r}=0.60$ compared with $\mathrm{r}=0.66$ in the full age range sample, it is still drastically higher than the $\mathrm{r}=0.30$ found in UKBB.

\section{Overlapping and unique variance in chronAge explained across omics clocks}

Interestingly, the proportion of unique variance in chronAge explained by each OCA does not entirely mirror the univariate $R^{2}$ (black dots) (Supplementary Figure 5B). It is important to note that the similarity between assays likely influences the proportion of unique variance in chronAge explained (at its most extreme, were a clock duplicated, it would explain no unique variance). This may explain why NMR metabolomics,
MetaboAge and MS complex lipidomics clocks have some of the lowest proportions of unique variance explained, despite NMR metabolomics and MS complex lipidomics having an $R^{2}$ higher than DEXA OCA and comparable to Clinomics. Interestingly, the DEXA and MS fatty acids lipidomics OCAs explain more unique variance than several clocks with higher univariate $R^{2}$. MetaboAge stands out with low unique variance paired with the lowest univariate $R^{2}$ in chronAge.

\section{Pairwise clock comparisons of variance explained in chronAge}

Partly to consider the effect of two similar clocks affecting the unique variance explained, we performed pairwise comparisons, the unique variance in chronAge explained by each clock in the comparison was again calculated as the squared part correlation while controlling for the other clock in the pair (Supplementary Table 6). The overlap indicated is therefore the proportion of variance in chronAge explained by both clocks in the pair. Reiterating the results in Supplementary Figures 5A, 6 shows that for 10 out of 14 clocks the mean percentage of variance explained in chronAge by both clocks (the overlap) is greater than 40\%. The MS Fatty Acids Lipidomics and DEXA clocks had lower mean overlap, $23.2 \%$ and $36.9 \%$ respectively, with MetaboAge the lowest mean overlap across clocks $3.6 \%$. Interestingly clocks that had higher correlations between OCA and chronAge, such as PEA Proteomics and DNAme-based clocks were found to be contributing most of the additional variance in chronAge not explained by the overlap of both clocks. Conversely, the MS Fatty Acids Lipidomics clock, the clock with the second lowest correlation between OCA and chronAge appears to contribute little of the additional variance in chronAge not already explained by the other clock across all comparisons. This is even more extreme for MetaboAge, in addition to extremely low average overlap in variance explained in chronAge across clocks, the other clock in the comparison contributes the majority of the variance explained. This observation, that the comparison with MetaboAge shows the lowest overlap, is consistent across all other clocks, including NMR Metabolomics which is derived from the same omics assay.

\section{Association of chronAge and OCAAs with health outcomes}

7/7 risk factors and 43/44 disease groupings associated with chronAge in the expected positive direction, except 
for cortisol and FEV1 which decline with chronAge (Supplementary Figure 7A, 7B). The disease exception, J00-J06 Acute respiratory infections, was not nominally significantly different from zero $\left(\log _{\mathrm{e}} \mathrm{HR} / \mathrm{SE}-0.025 /\right.$ 0.017). All the risk factors, and 34 of the disease grouping associations were significant after allowing for multiple testing (passed FDR 10\% risk factors and diseases considered separately, one sided test $\mathrm{H}_{1}: \mathrm{b}>0$ ). For these 34 groupings there thus was reasonable power to detect associations with chronAge and so potentially biological OCAA. 2 disease groups had fewer than 5 cases and were excluded from the subsequent analysis, to further limit the burden of multiple testing.

The effect $\left(\log _{\mathrm{e}} \mathrm{HR} / \mathrm{SE}\right)$ of one year of chronAge at outset on the first incidence of any of the diseases was $0.0492 / 0.00323$, a doubling roughly every 14 years. This pattern was generally similar to the estimated effects for each disease individually, noting these are on the same (logistic) scale. With the largest observed differences arising from diseases with larger standard errors. However, the effect $\left(\log _{\mathrm{e}} \mathrm{HR} / \mathrm{SE}\right)$ of one year of chronAge on the risk factors varied more, although again they were on the same (standardised) scale. FEV1 and systolic BP (-0.041/0.00088 and 0.035/0.0015) were most sensitive, whilst CRP and creatinine were less sensitive (effect/SE of 1 year of chronAge on standardised trait 0.0092/0.0012 and 0.0090/0.0015 respectively) as shown in Supplementary Figure 7B, whilst standard errors of the effect sizes were generally smaller (as a proportion of the effect).

\section{Assessment of smoking as a potential confounder}

Across all the associations studied for 15 clocks against 32 diseases and 7 risk factors, we found that the IVW ratio of the estimated effect of OCAA with and without smoking fitted as a covariate were 1.012 and 1.011 respectively. Individual test $\mathrm{p}$-values for the ratio of the effects not being one all exceeded 0.35 . Visual analysis confirmed these results: that smoking was not a material confounder of health-OCAA associations. 


\section{Supplementary Figures}

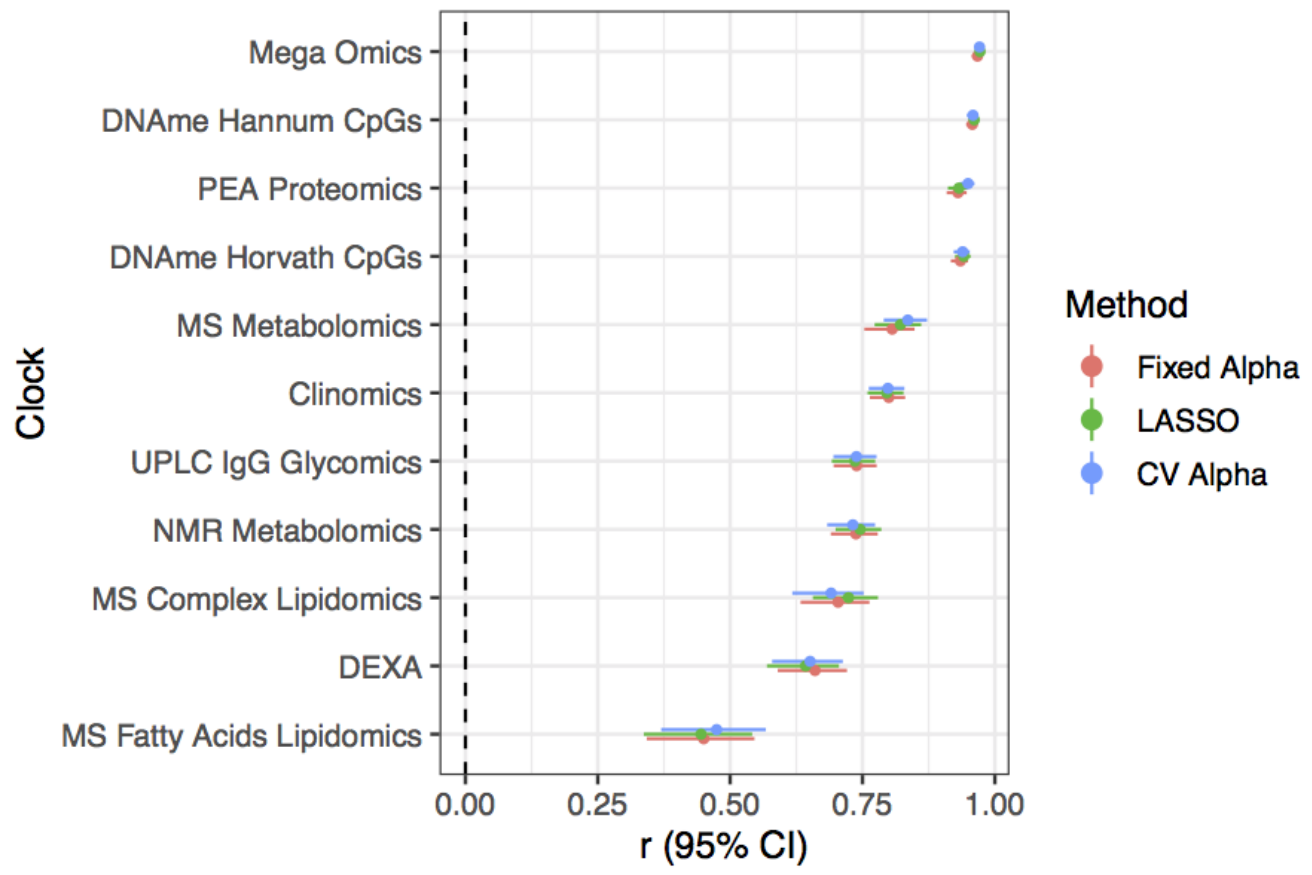

Supplementary Figure 1. Correlation of chronAge and OCA were consistent, independent of penalised regression method. Correlation ( $r$ ) with $95 \%$ of confidence intervals of chronAge with omics clock estimated age (OCA) indicated on the $y$-axis via elastic net regression with a fixed alpha of 0.5 , cross validated alpha and LASSO regression in the ORCADES testing sample.

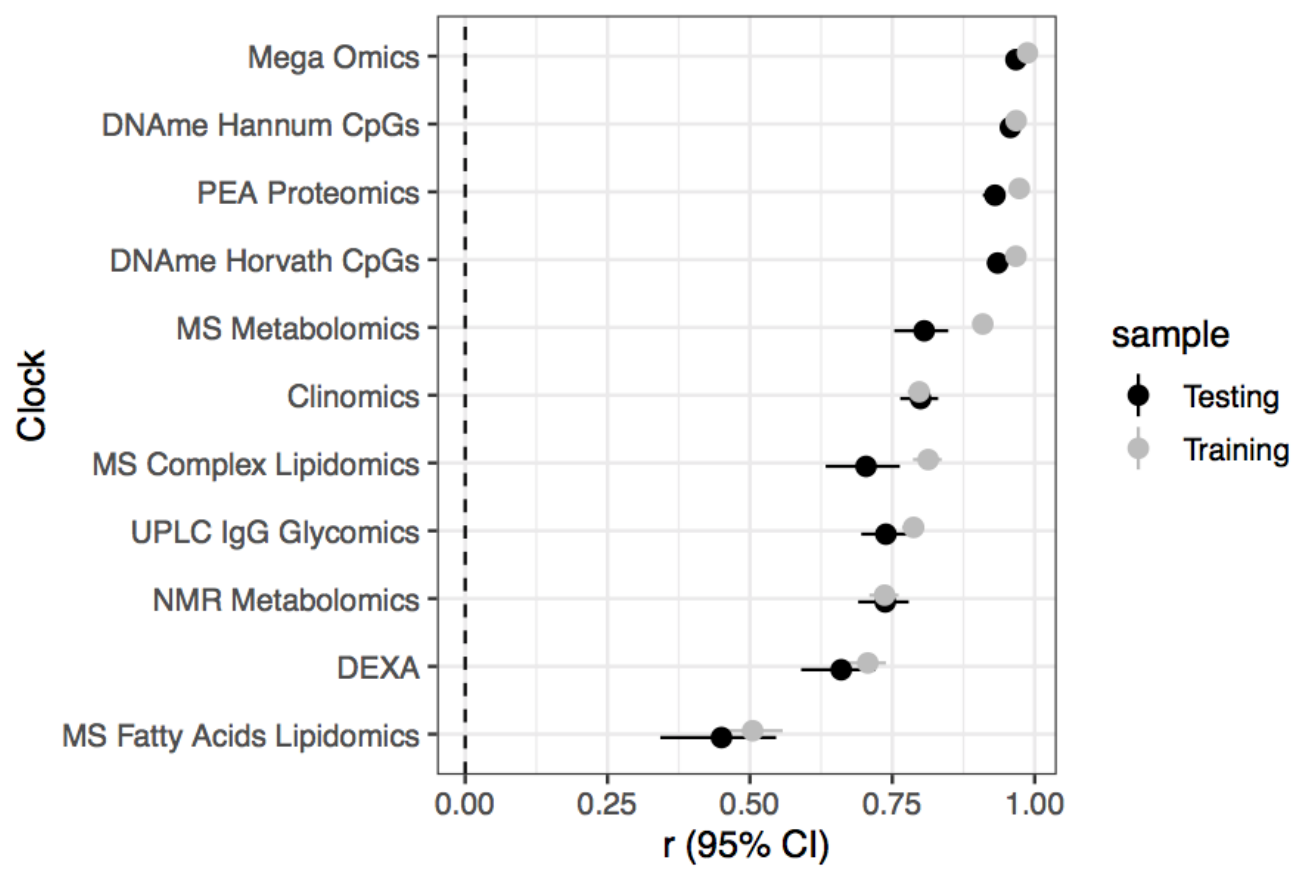

Supplementary Figure 2. Correlation of chronAge and OCA in ORCADES training and testing samples. Correlation ( $r$ ) with 95\% of confidence intervals of chronAge with OCA indicated on the $y$-axis in the ORCADES Training and Testing samples. 

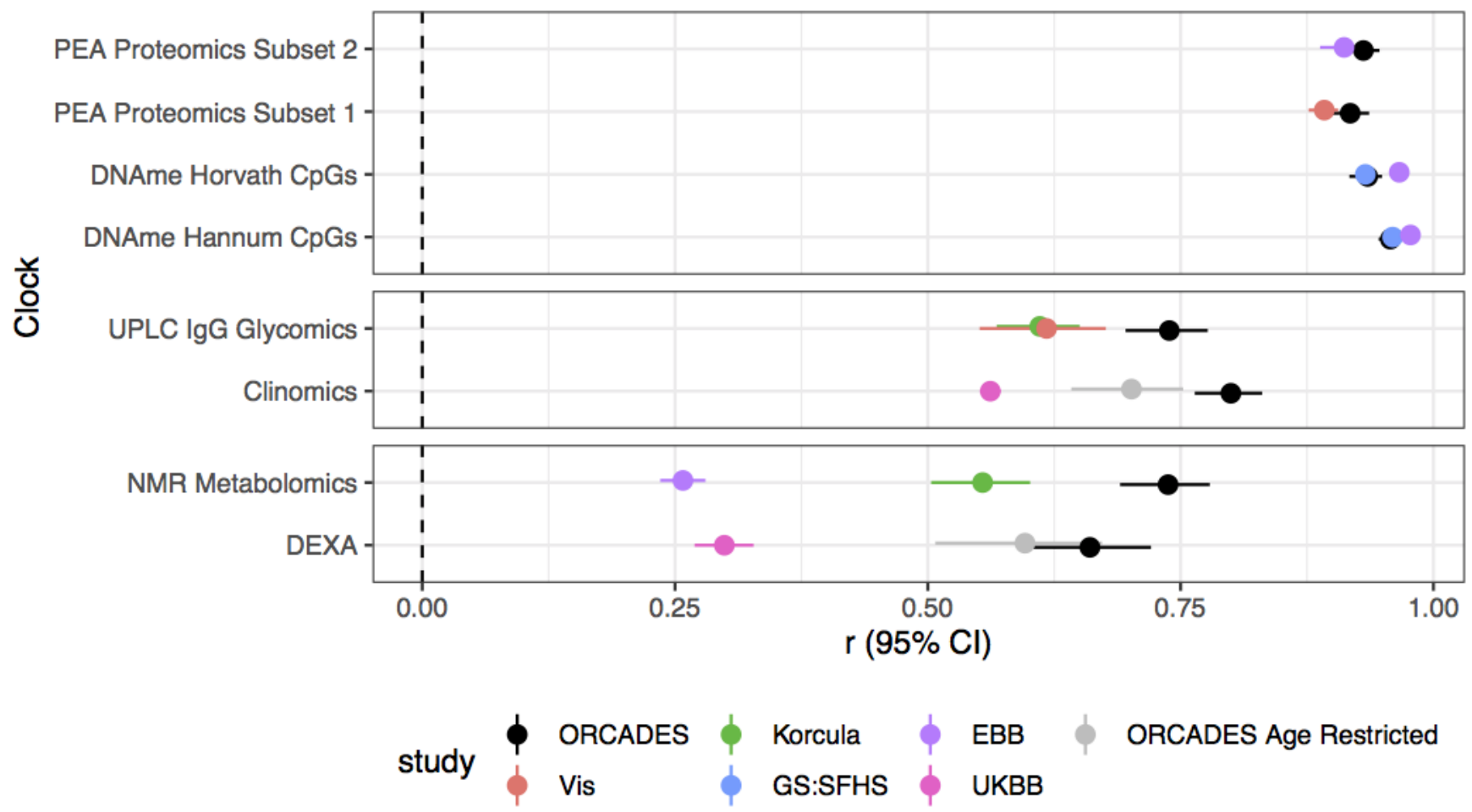

Supplementary Figure 3. Omics clocks trained in ORCADES predict chronAge in unrelated cohorts. The correlation of OCA with ChronAge ( $\mathrm{x}$-axis) by the specified clock ( $\mathrm{y}$-axis). With the correlation in the ORCADES testing sample in black and additional populations as specified. The correlation in a restricted age range (40-75) ORCADES testing sample is shown in comparisons involving the UKBB shown in grey.

\section{Cluster Dendrogram}

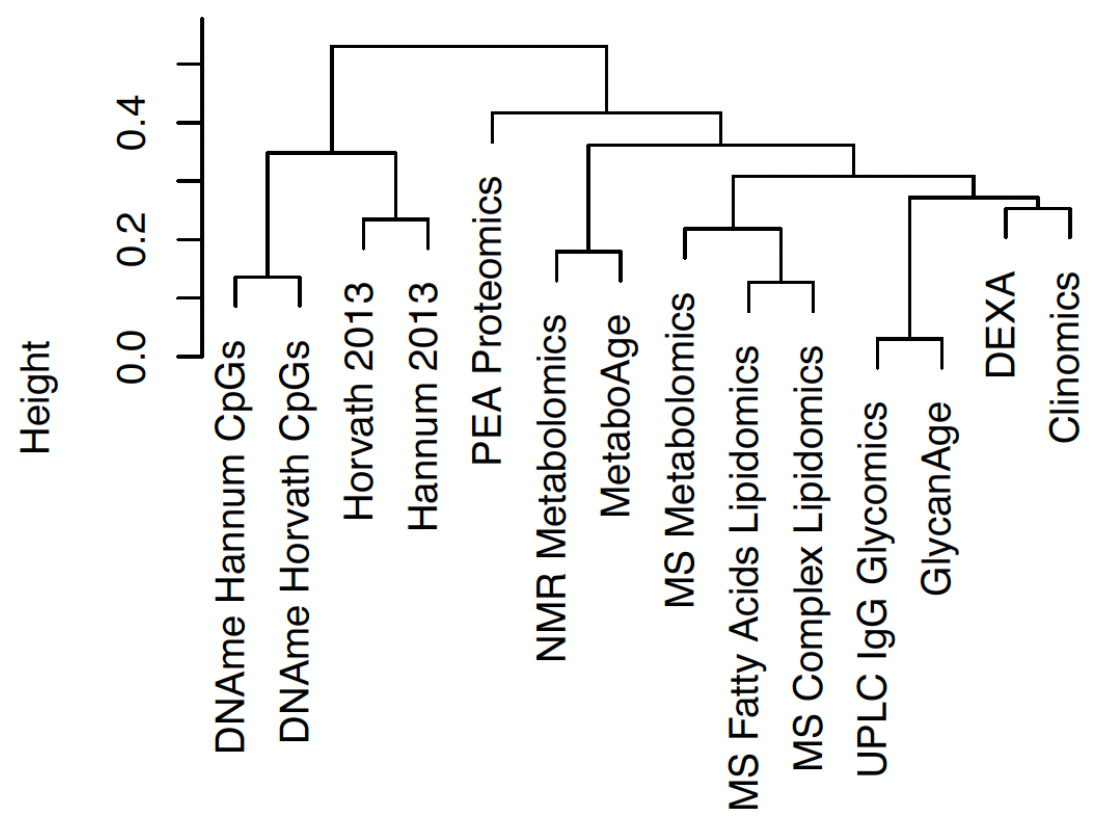

Supplementary Figure 4. Dendrogram of omics age acceleration measures. Based on hierarchical clustering of OCAA measures from each clock. Mega-Omics was excluded from this analysis as it contains predictors from multiple omics assays. 
A
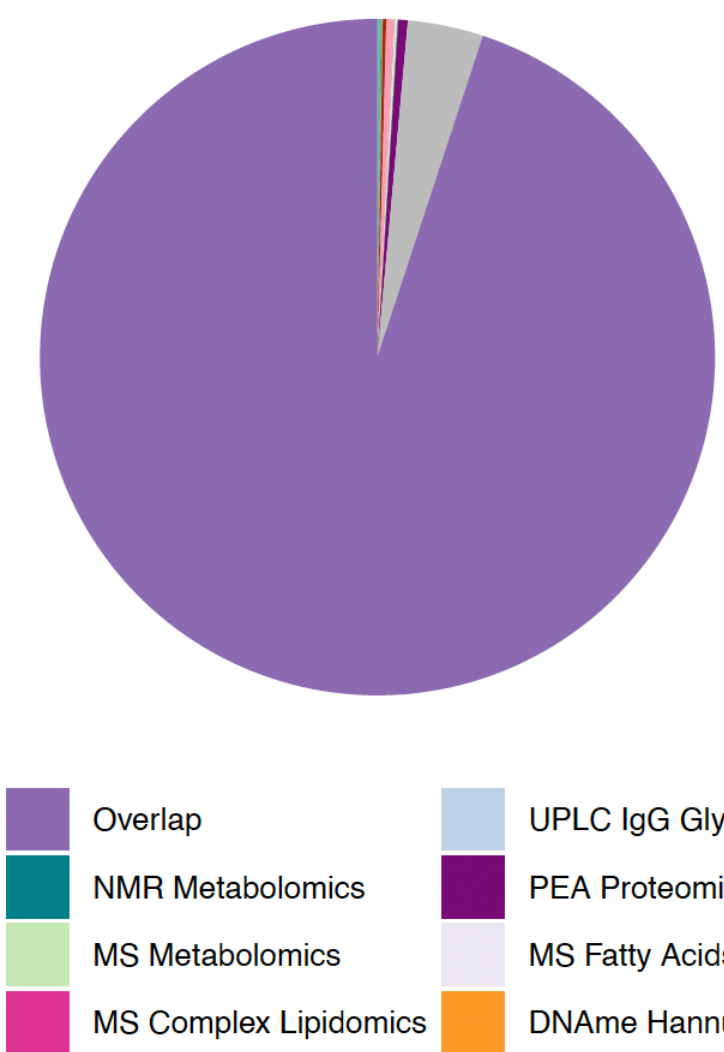

Overlap

NMR Metabolomics

MS Metabolomics

MS Complex Lipidomics

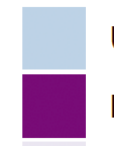

B

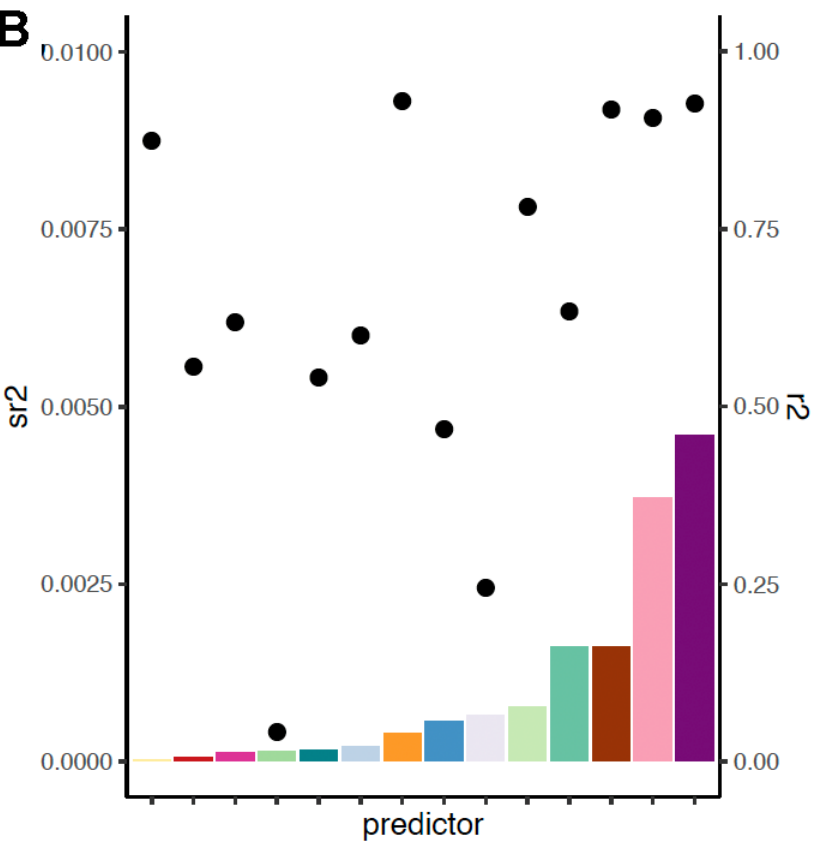

UPLC IgG Glycomics

PEA Proteomics

MS Fatty Acids Lipidomics

DNAme Hannum CpGs

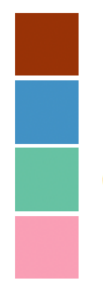

DNAme Horvath CpGs

DEXA

Clinomics

Hannum 2013
Horvath 2013

MetaboAge

GlycanAge

Unexplained

Supplementary Figure 5. Overlapping and unique variance in chronAge explained across 10 omics clocks. (A) Partition of variance in ChronAge explained into that explained by 2 or more clocks (overlap), that not explained by any clock (unexplained), and that explained by each of the 10 clocks uniquely. Segments coloured by component explaining the variance in chronAge. (B) squared part correlations $\left(\mathrm{sr}^{2}\right)$ (bars): unique variance in chronAge explained by each of the 10 clocks from Figure $A$ on the left-hand $y$-axis. $R^{2}$ (points) indicate the total variance explained in chronAge by each clock (right hand $y$-axis). 


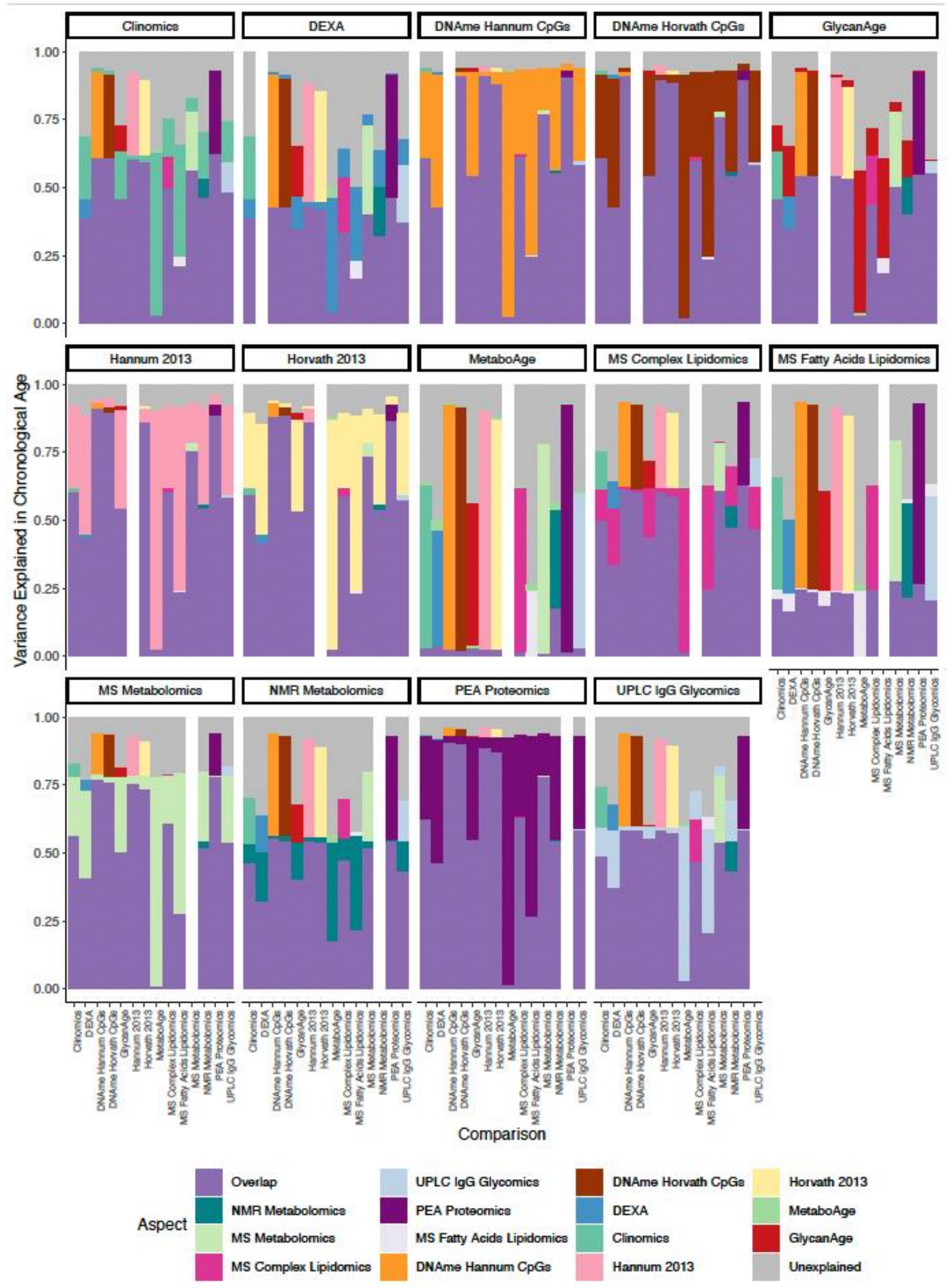

Supplementary Figure 6. Pairwise comparisons of variance explained in chronAge. Pairwise comparison of variance in chronAge explained by OCA of the pairs of clocks in ORCADES. Comparison indicated on the x-axis, with the variance in chronAge explained on the $y$ axis. The colour of the bar indicates the aspect explaining the variance. For each comparison the proportion of variance explained by both clocks in the comparison (Overlap), the variance that remains unexplained fitting a bivariate model (unexplained) and the unique variance in chronAge explained by each of the two clocks in the comparison. 


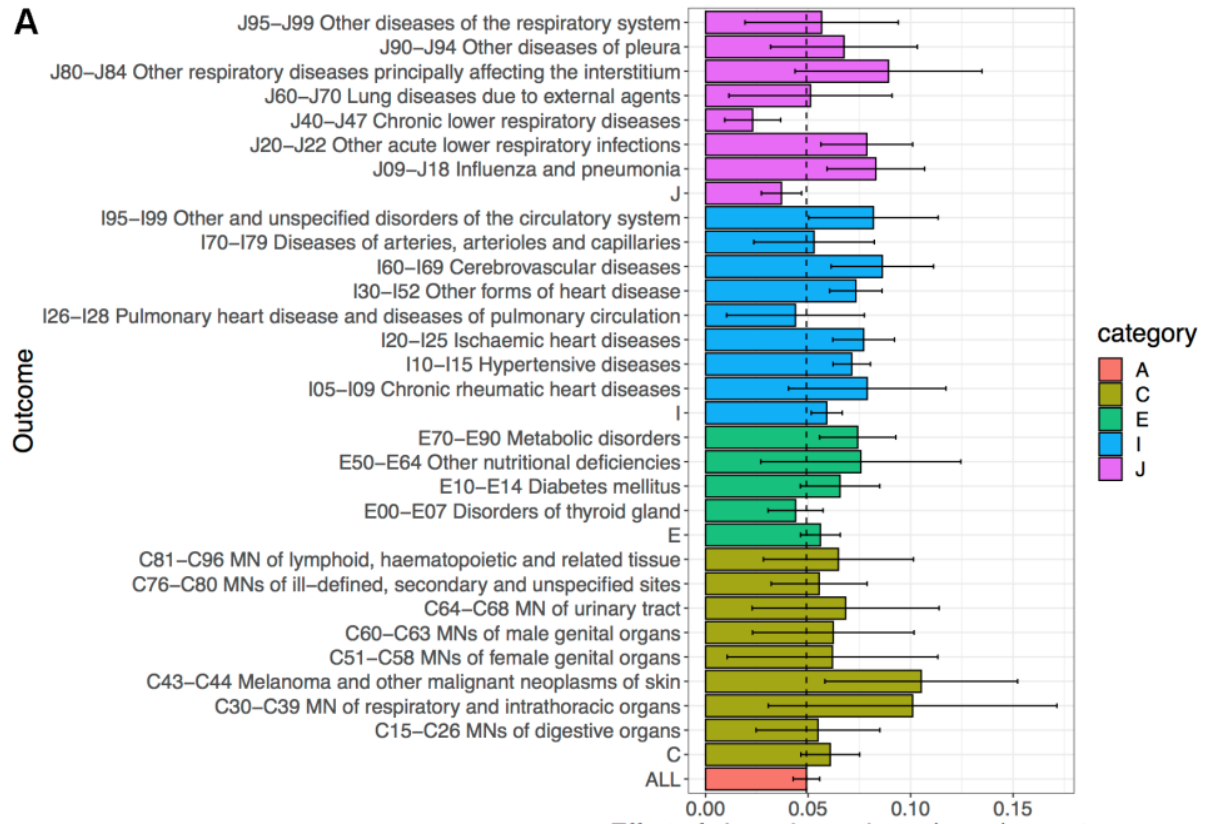

Effect of chronologocal age (years) on outcome

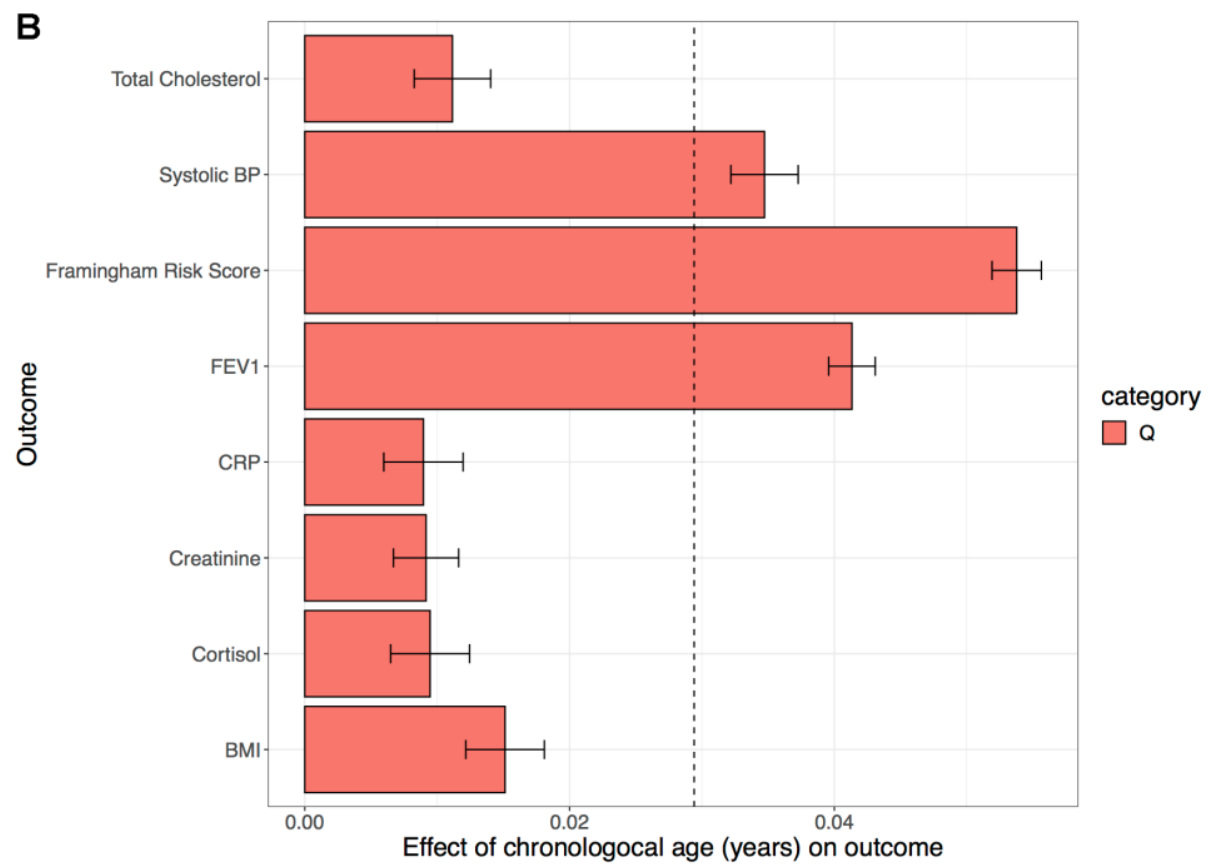

Supplementary Figure 7. Associations with disease incidence. (A) Associations of disease incidence with chronAge. Effect and its $95 \%$ $\mathrm{Cl}$ : the $\log _{\mathrm{e}} \mathrm{HR}$ of chronAge on the incidence of the disease since participation, using a Cox Model. ICD 10 Chapters (i.e. whole Categories) count the first occurrence (post assessment) of any disease within the letter/category/chapter (including those blocks dropped from the individual block analysis due to lack of power) as incidence. Participants prevalent at assessment (i.e. a recorded prior incidence) within any grouping at assessment were excluded from the analysis of that grouping. The dashed line represents the hazard of age on any occurrence of the disease chapters under consideration, a hazard ratio of 0.0492 , representing a doubling of incidence rate every 14 years. Distinctions in observed individual effects sizes from this were (visually) judged more materially due to sampling variance than true effects, and so that single factor was chosen as our best estimate of the age effect on each disease. MNs: Malignant neoplasms. Associations are only shown for those disease groups that passed QC and were taken forward to association testing with OCAA. (B) The strength of associations of risk factors with chronAge varies. FEV1: Forced expiratory volume one second, CRP: C-reactive Protein, BMI: Body Mass Index. Effect: the estimated increase (and $95 \% \mathrm{Cl}$ ) in standardised trait per year of chronAge using a linear model, with sex as a covariate. Traits which decrease as age increases (FEV1, cortisol) have been converted to ageing traits, by reversing their signs. 

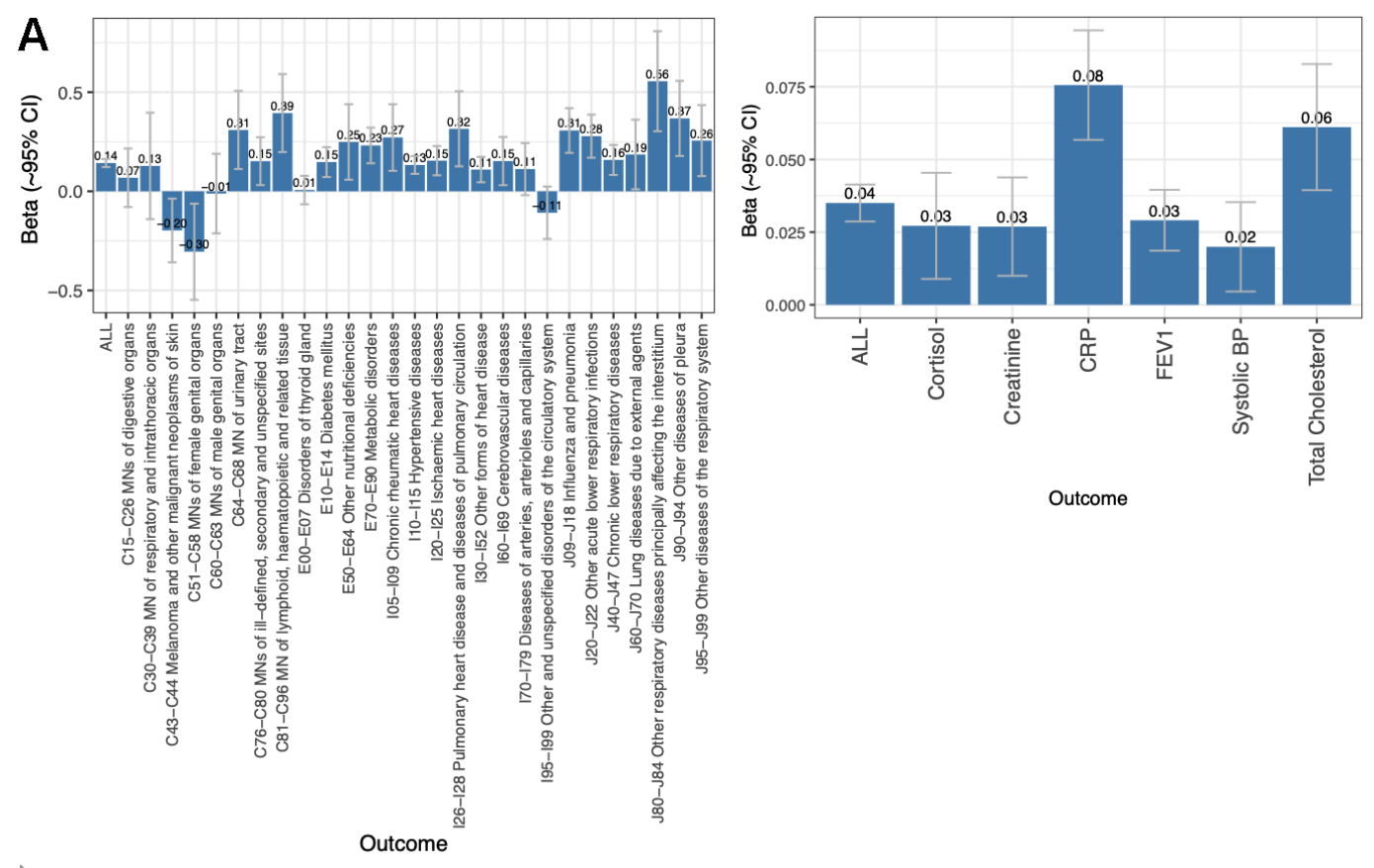

B

Risk Factors
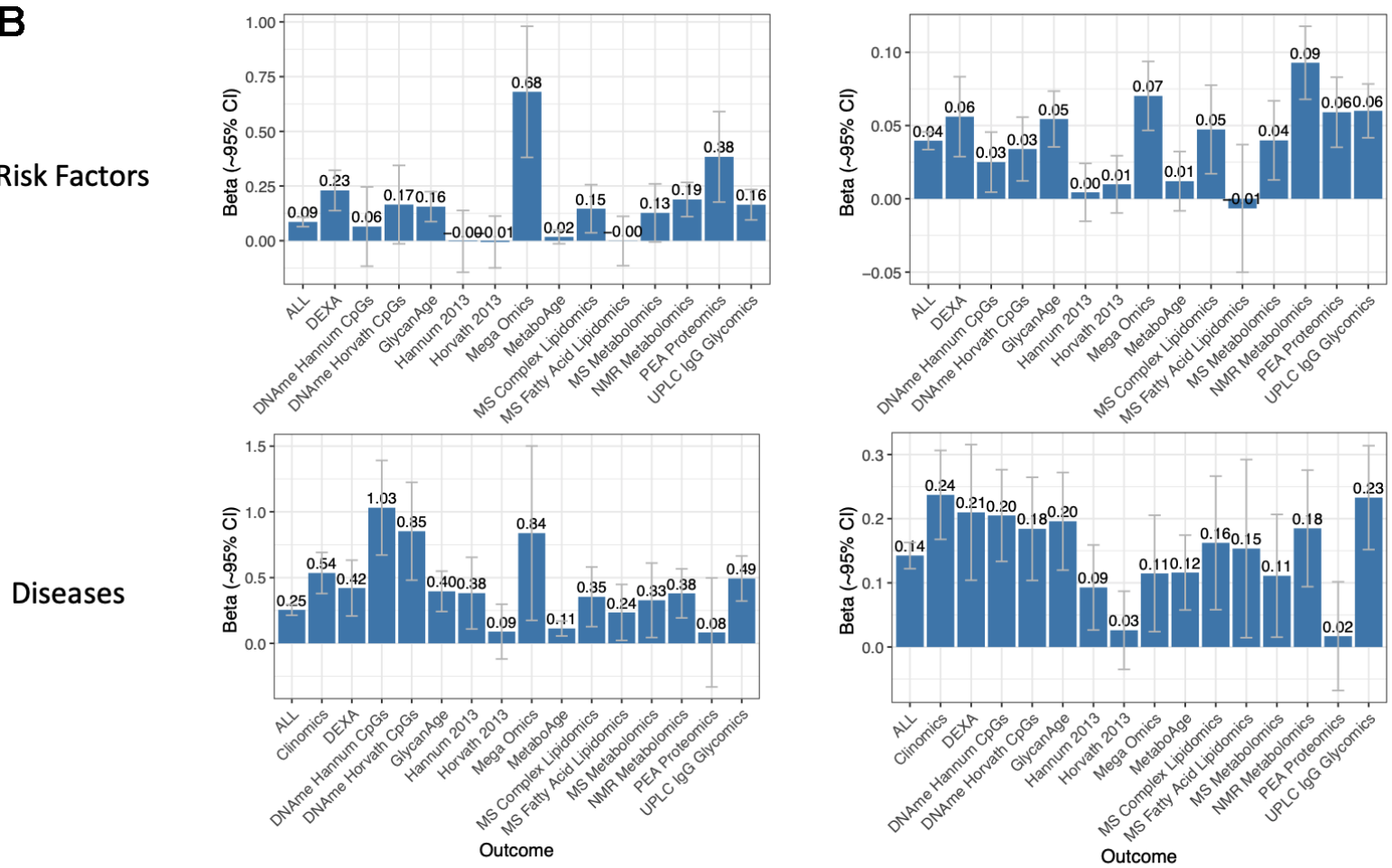

OCAA (years) effect per year
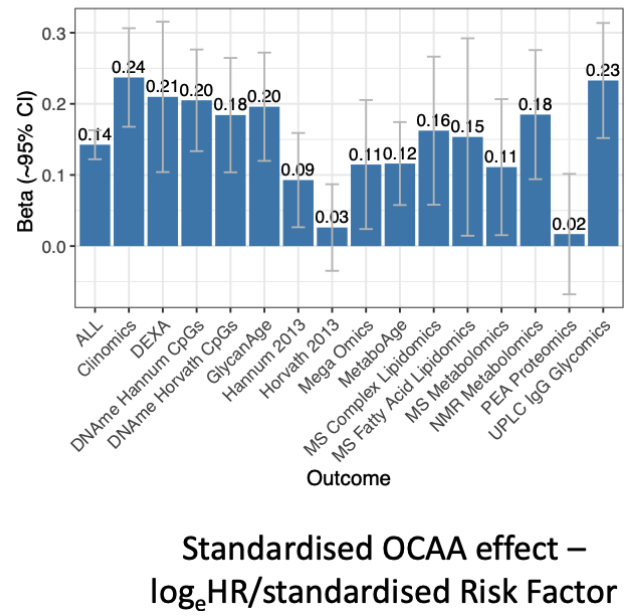

Supplementary Figure 8. (A) Average effect across clocks of standardised OCAA upon outcome. Beta: the observed effect of OCAAs on outcome. Beta was IVW averaged across OCAAs. SEs were calculated as the inverse root sum of the precisions (not strictly valid given correlated tests). Error bars shown are \pm 2 SEs. OCAA: omics clock estimated age acceleration. (B) Averaged effects of OCAA across diseases and risk factors. The Left-hand side shows the effect of OCAA in years per year of chronAge effect (OCAA effect divided by chronAge effect) IVW averaged across outcomes (either risk factors or diseases as specified on the y-axis). The right-hand side shows the effect of standardised OCAA (units of phenotypic standard deviation) IVW averaged across outcomes. Beta: the observed effect of OCAA on outcome. Beta was IVW averaged across outcomes. SEs were calculated as the inverse root sum of the precisions (not strictly valid given correlated tests). Error bars shown are $\pm 2 S E s$. OCAA: omics clock estimated age acceleration. 


\section{A Age accelaration}

not mediated through smoker status

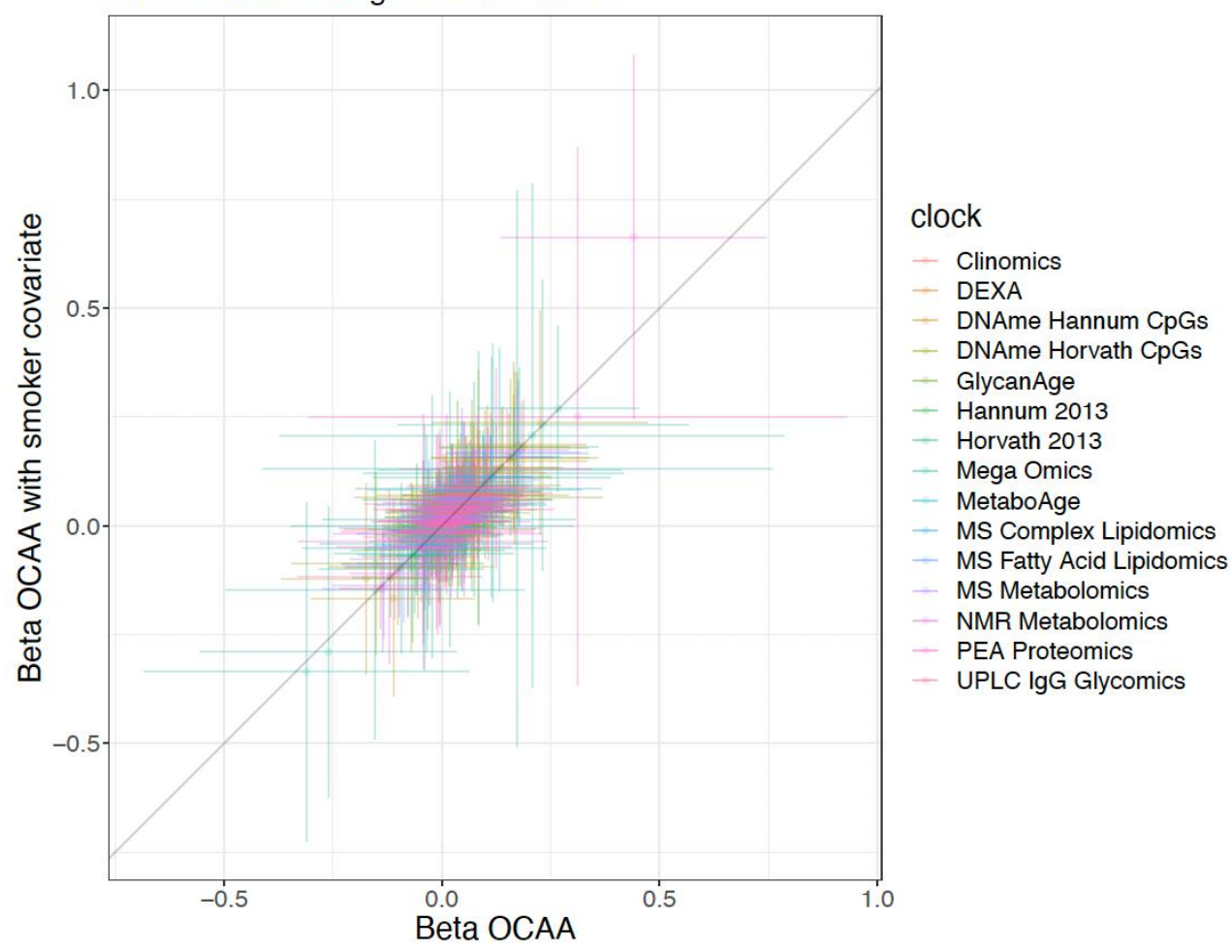

B Age accelaration

not mediated through smoker status

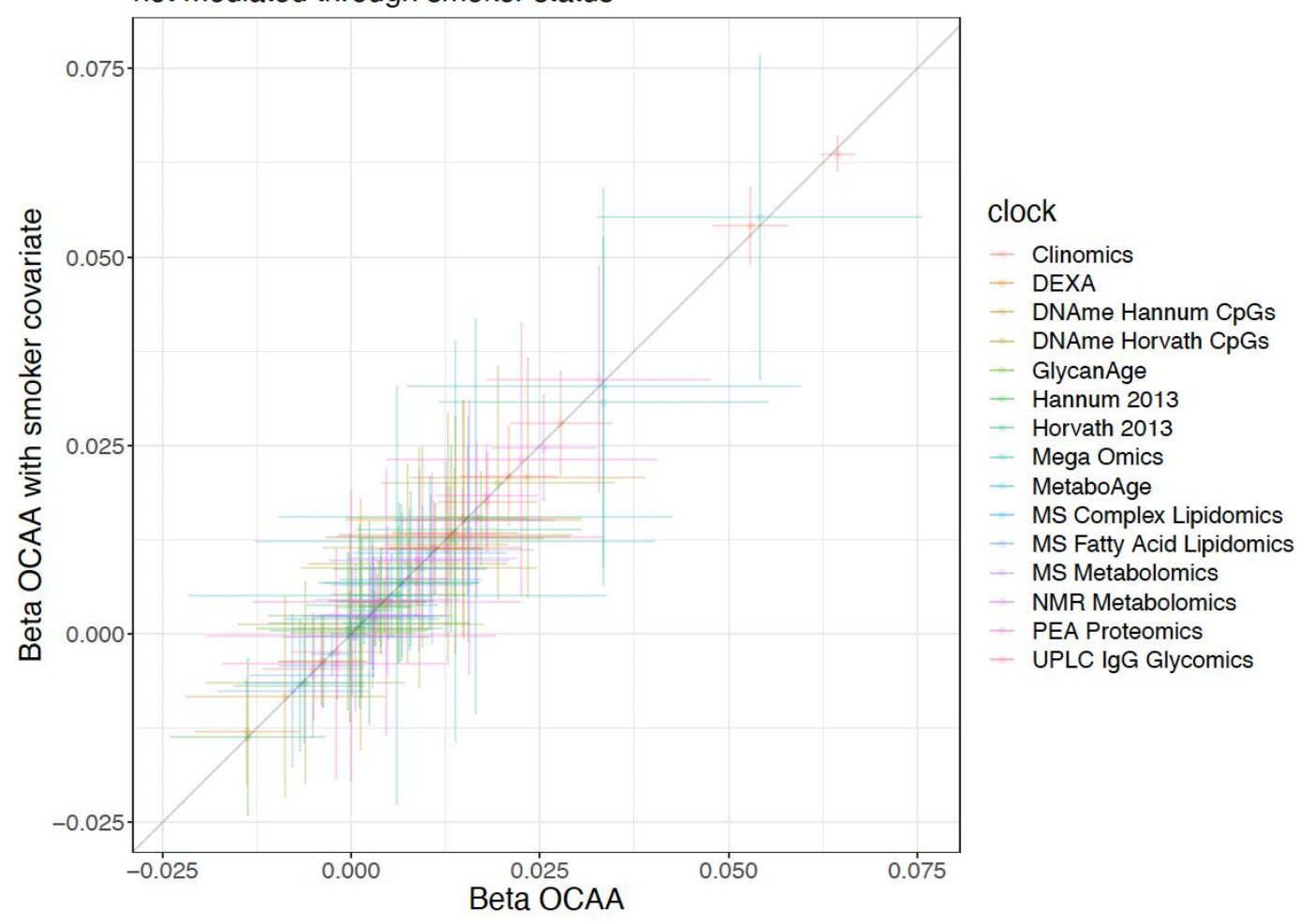

Supplementary Figure 9. Fitting smoking as a covariate does not appear to materially affect the association between OCAA and (A) diseases or (B) risk factors. Beta OCAA - the observed effect of OCAA on the outcome under the models (see main text). Beta OCAA with smoker covariate - the observed effect of OCAA on the outcome under the same model, but with smoking fitted. 

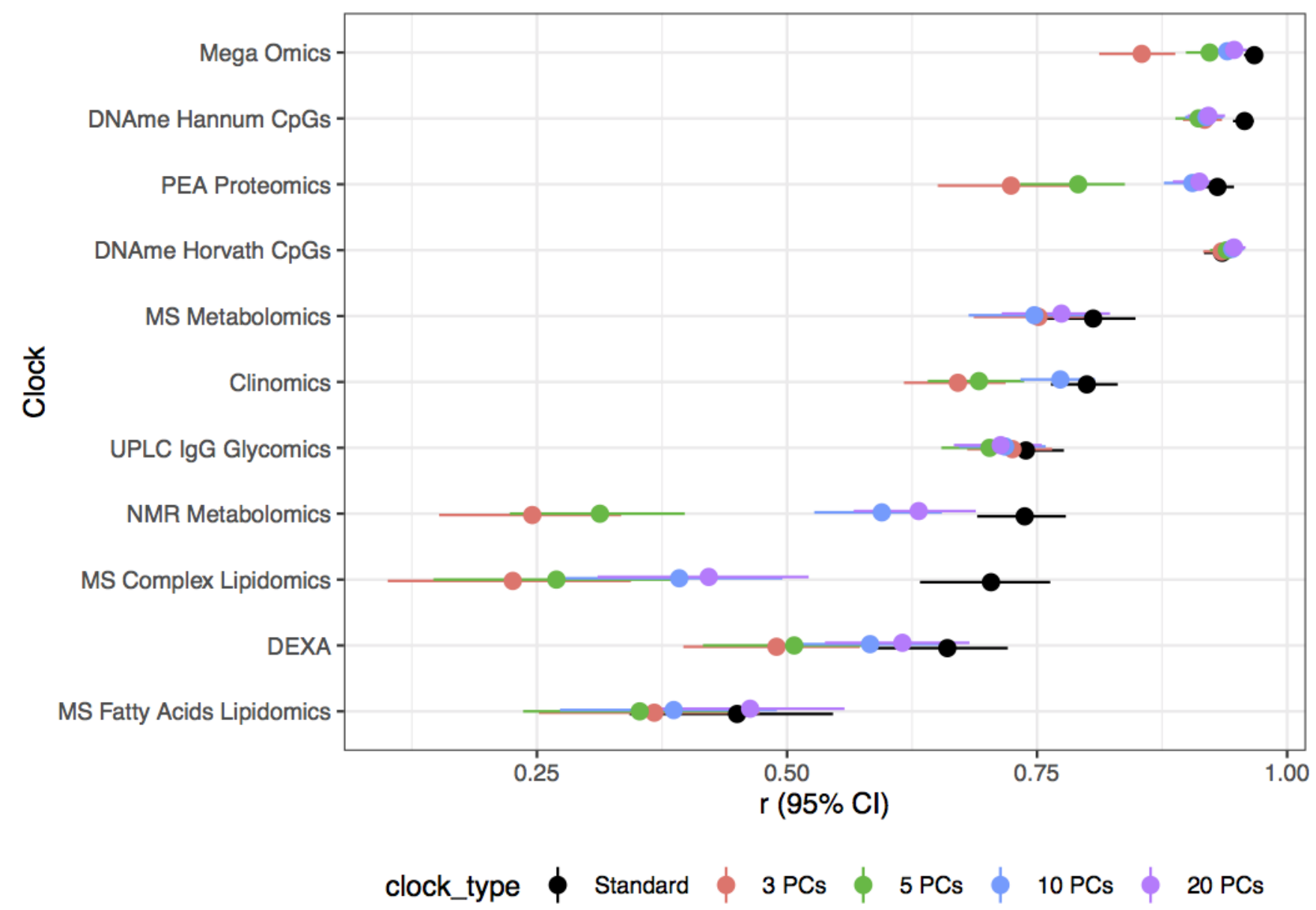

Supplementary Figure 10. Correlation of chronAge and OCA from clocks built using 3, 5, 10, 20 PCs. Correlation (r) and 95\% confidence interval of chronAge and OCA indicated on the $y$-axis using models constructed from 3, 5, 10 and 20 principal components of the assay in the ORCADES testing sample compared to the standard clock (black). 

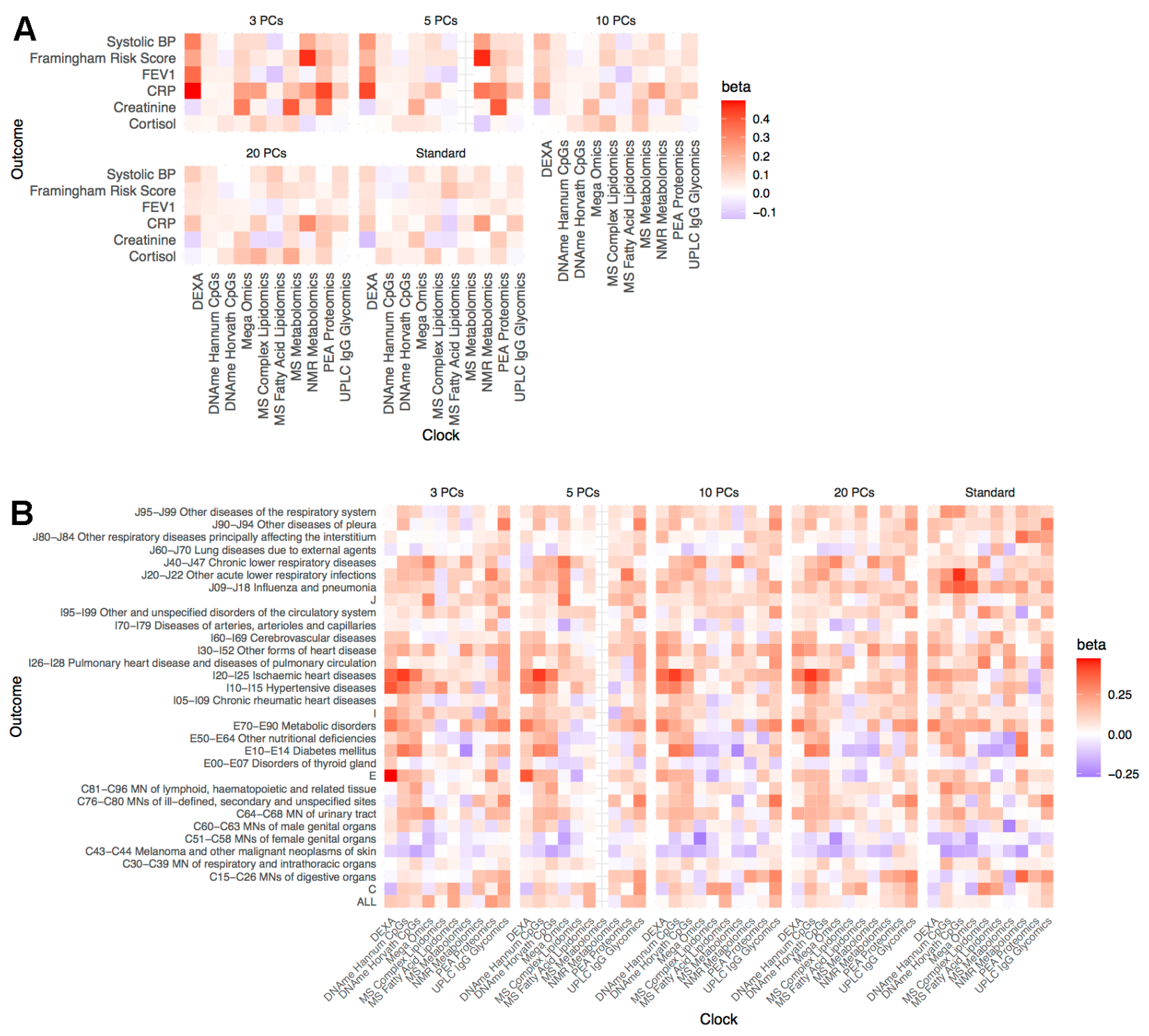

Supplementary Figure 11. (A) Reducing dimensionality of omics dataset used to build clocks increases the predictive ability of OCAA for risk factors. Beta: the effect of a year of standardised (within clock) OCAA on outcome (effect sizes for standardised risk factors). Estimates were shrunk using a prior to reduce the possibility that frequentist best estimate beta was predominantly a consequence of a large SE. Clock: the omics clock on which OCAA was measured. Cholesterol/BMI which showed a particularly large effect from MS Fatty Acids Lipidomics/DEXA OCAA, excluded here to aid visualisation. X PCs: the number of PCs of the omic used as predictors to create the chronAge and OCAA measures. Clinomics was excluded from this analysis as it was based on only 12 predictors. (B) Reducing dimensionality of omics dataset to train ChronAge makes little difference to the predictive ability of OCAA for diseases. Beta: the effect of a year of standardised (within clock) OCAA on outcome (measured in $\log _{e}$ hazard ratios). Estimates were shrunk using a prior to reduce the possibility that frequentist best estimate beta was predominantly a consequence of a large SE. Clock: the omics clock on which OCAA was measured. Disease group: the set of diseases (defined by ICD 10 codes) which were tested for first incidence after assessment against the clock (already prevalent cases were excluded). X PCs: the number of PCs of the omic used as predictors to create the chronAge and OCAA measures. Clinomics was excluded from this analysis as it was based on only 12 predictors. 


\section{Supplementary Tables}

Please browse Full Text version to see the data of Supplementary Tables 2, 3, 5, 6 .

Supplementary Table 1. ORCADES descriptive statistics.

\begin{tabular}{lcccccc}
\hline Omic & $\mathbf{N}$ & Mean age & SD age & Min age & Max age & \% Female \\
\hline DEXA & 1158 & 55.85 & 14.19 & 18.02 & 88 & 59.93 \\
DNAme Horvath CpGs & 957 & 52.93 & 15.66 & 17.12 & 100.18 & 55.38 \\
MS Fatty Acids Lipidomics & 952 & 53.41 & 15.49 & 16.84 & 91.47 & 55.78 \\
MS Metabolomics & 861 & 52.81 & 15.05 & 17.12 & 90.79 & 57.38 \\
Clinomics & 1815 & 53.35 & 15.03 & 16.5 & 91.47 & 59.56 \\
DNAme Hannum CpGs & 1033 & 53.43 & 15.68 & 17.12 & 100.18 & 55.86 \\
UPLC IgG Glycomics & 1937 & 53.13 & 15.29 & 16.5 & 100.18 & 60.51 \\
MS Complex Lipidomics & 940 & 53.54 & 15.27 & 17.12 & 91.47 & 55.74 \\
NMR Metabolomics & 1643 & 52.96 & 14.91 & 16.5 & 91.47 & 59.95 \\
PEA Proteomics & 805 & 52.88 & 15.59 & 17.12 & 91.47 & 54.91 \\
Mega Omics & 796 & 53.1 & 15.31 & 17.12 & 91.47 & 56.78 \\
GlycanAge & 1957 & 53.15 & 15.3 & 16.5 & 100.18 & 60.4 \\
MetaboAge & 1947 & 53.07 & 15.33 & 16.5 & 100.18 & 60.45 \\
Hannum 2013 & 1052 & 53.51 & 15.75 & 17.12 & 100.18 & 55.8 \\
Horvath 2013 & 1052 & 53.51 & 15.75 & 17.12 & 100.18 & 55.8 \\
\hline
\end{tabular}

Omic: Omic assay.

$\mathrm{N}$ : number of individuals in ORCADES with the omics assay passing quality control.

Mean Age: mean chronological age at venepuncture of ORCADES subset.

SD Age: standard deviation of chronological age at venepuncture of ORCADES subset.

Minimum Age: minimum chronological age at venepuncture of ORCADES subset.

Maximum Age: maximum chronological age at venepuncture of ORCADES subset.

$\%$ Female: percentage of ORCADES subset that is female.

Supplementary Table 2. Description of biomarkers in ORCADES.

Supplementary Table 3. Coefficients for clocks trained in ORCADES. 
Supplementary Table 4. ICD10 definitions.

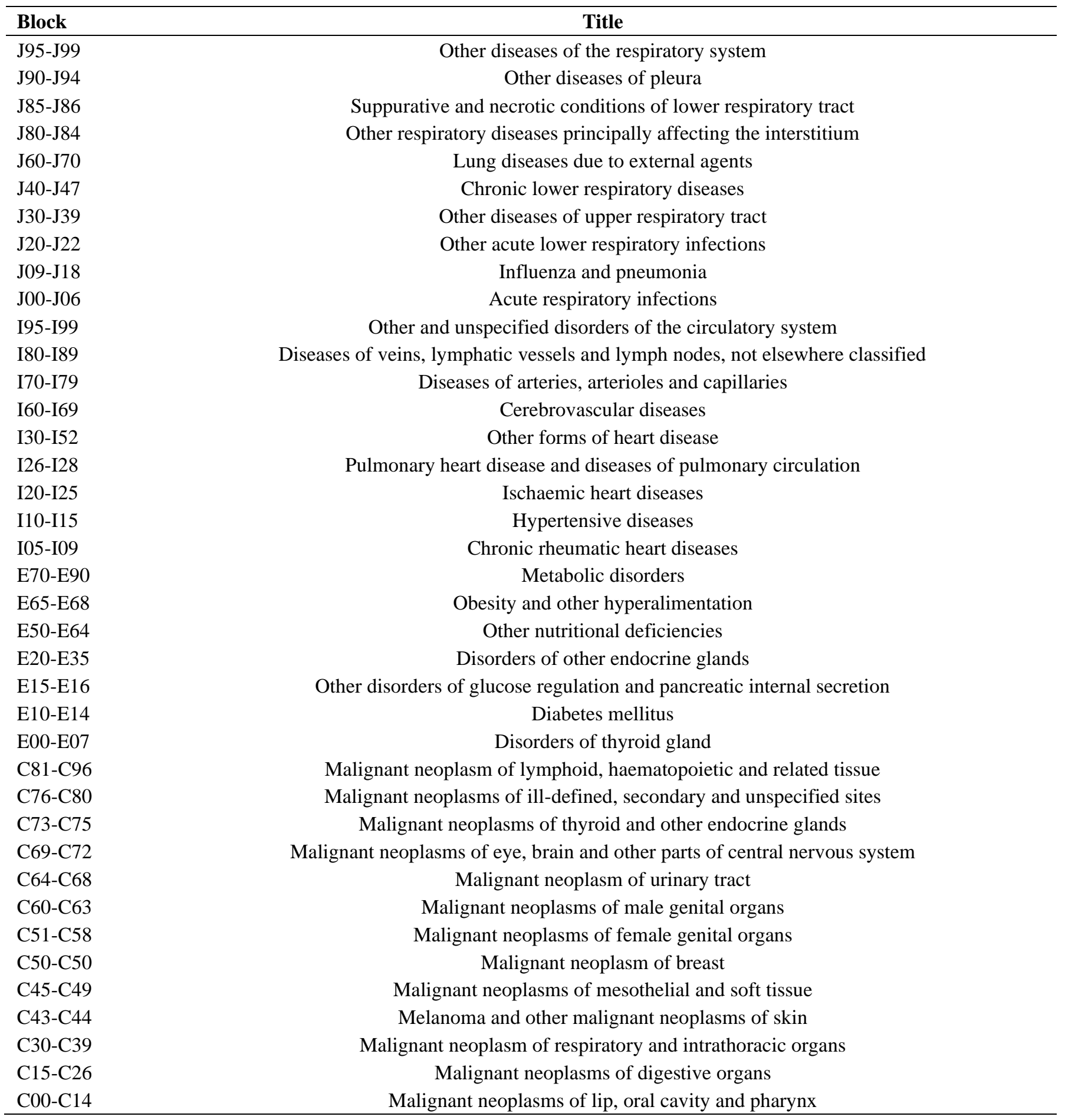


Supplementary Table 5. Summary of the association test between outcome and OCAA.

Supplementary Table 6. Sex stratified standardised OCAA-disease block associations. 